Article

\title{
Stage-Dependent Structured Discrete-Time Models for Mosquito Population Evolution with Survivability: Solution Properties, Equilibrium Points, Oscillations, and Population Feedback Controls
}

\author{
Manuel De la Sen ${ }^{1, *(\mathbb{D}}$, Asier Ibeas ${ }^{2}(\mathbb{D})$ and Aitor J. Garrido ${ }^{3}$ \\ 1 Institute of Research and Development of Processes IIDP, University of the Basque Country, Campus of \\ Leioa, Barrio Sarriena, 48940 Leioa, Bizkaia, Spain \\ 2 Department of Telecommunications and Systems Engineering, Universitat Autònoma de Barcelona, \\ Campus de Bellaterra, Bellaterra, Cerdanyola del Vallès, 08193 Barcelona, Spain; asier.ibeas@uab.cat \\ 3 Department of Automatic Control and Systems Engineering, Faculty of Engineering of Bilbao, University of \\ the Basque Country, Paseo Rafael Moreno 3, 48013 Bilbao, Spain; aitor.garrido@ehu.eus \\ * Correspondence: manuel.delasen@ehu.eus
}

Received: 11 November 2019; Accepted: 29 November 2019; Published: 3 December 2019

check for updates

\begin{abstract}
This paper relied on the investigation of the properties of the stage-structured model of coupled larvae and adult mosquito populations' evolution when parameterized, in general, by time-varying (or stage-dependent) sequences. In particular, the investigated properties were the non-negativity of the solution under non-negative initial conditions, the boundedness of the sequence solutions under any finite non-negative initial conditions, the equilibrium points, and the convergence conditions to them in the event that the parameterizing sequences converge to finite limits. Some further properties that were investigated relied on deriving the oscillation conditions of the solutions under certain conditions of the parameterizations. The use of feedback controls to decrease the foreseen numbers of alive mosquitoes in future evolution stages is also proposed. The proposed control actions are exerted on the birth rate and/or the maximum progression rate sequences. Some illustrative examples are also given.
\end{abstract}

Keywords: equilibrium points; Beverton-Holt equation; mosquito evolution solution stages; population controls; asymptotic stability; stability; oscillatory solutions

\section{Introduction}

It is well-known from the related background literature that mosquitoes undergo complete metamorphosis following a life cycle of four stages, namely, egg, pupae, larva, and adult. See, for instance, [1] for an easily comprehensible description of those stages and the duration period of each of them. Mosquitoes typically stay in an aquatic environment during the first three life stages and in an aerial environment during their adult stage. Mathematical models are useful to describe the evolution of the mosquito population. The introduction of sterile mosquitoes in the environment in order to reduce the fertility of the whole population and control their populations under acceptable levels of tolerance has been proposed in the background literature. See, for instance, [1-3], and some of the references therein. Also, the study of releases of sterile mosquitoes has been investigated in [4], with a Beverton-Holt type model for survivability, as well as in [5]. It is well-known that Beverton-Holt type models are very popular to study the evolution of species that reproduce by eggs, [4,6-12] such as 
birds, insects, or fish. More general difference equations which generalize those kinds of problems are studied in detail in $[13,14]$.

The investigation of some evolution models for mosquitoes is directly linked to the evolution of some transmission diseases of host/vector type. For instance, in [5], a malaria model was studied with a stage-type evolution type of mosquitoes. See also some references therein and [12]. In [15-21] several epidemic models, without or with different vaccination and treatment controls of constant and feedback-type, were discussed and studied in detail. Note that, as far as our current problem at hand is concerned, it turns out that the use of programmed aerial or aquatic insecticide can also be of practical interest to control the mosquito populations as a potential alternative to the introduction of sterile mosquitoes in the habitat of wild mosquitoes. At the same time, it appears to be evident that some admissibility levels should be imposed on such controls in order to avoid intolerable ecological damage to the environment.

The paper discusses the properties of the stage-structured model of combined larvae and adult mosquito populations' evolution when parameterized by time-varying (or stage-dependent) sequences. In particular, the investigated properties were the non-negativity of the solution under non-negative initial conditions, the boundedness of the sequence solutions under finite non-negative initial conditions, the equilibrium points, and the convergence conditions to them if the parameterizing sequences converge to limits. Some further studied properties are referred to as the oscillation conditions of the solutions. The use of control to decrease the foreseen numbers of mosquitoes in future stages is also proposed. The control actions can be exerted on the birth rate and/or the maximum progression rate sequences. Physically, such controls can be the use of aquatic or aerial insecticide for the larvae and adults, respectively, or the introduction of sterile mosquitoes in the natural environment of wild mosquitoes. Those actions translate into modifying, "ad hoc", some of the parameterizing sequences appearing in the model. Some examples are also proposed and discussed in light of the theoretical modeling developments. Conditions for boundedness and exponentially fast convergence to the equilibrium point were also investigated. The evolution of both larvae and adult stages through time was also interpreted in the context of a derived artificial Beverton-Holt equation for each of the two evolution sequences. Each of those mentioned artificial or Beverton-like equations capture the necessary information about couplings with the other stage, that is the larvae influence in the adult sequence evolution and vice versa, which allows us to get additional results on the properties of the evolution sequences. This translates, in practice, into an additional presence of a one-step discrete delay in each Beverton-like equation, which is not present in the standard Beverton-Holt equations involving only one population, which is reflected in the structure of the environment's carrying capacity. Illustrative examples are also discussed.

The paper is organized as follows. Section 2 states the proposed model and describes and proves its main basic properties, namely, is positivity under non-negative initial conditions, as well as its stability and boundedness and convergence of the solution trajectory sequence. It also gives some illustrative examples. The section is split into three subsections to facilitate its reading, namely, the model and its well-posedness according to the positivity of the solution, its boundedness, stability and convergence, and some worked examples. In particular, it is discussed how the combination of both stages of larvae and adult can lead to the interpretation of the evolution model by an extended Beverton-Holt [6,9-11] equation with varying population-dependent carrying capacity. In particular, the model is equivalently reformulated by absorbing the mutual larvae/adult stage-couplings into the self-evolution of just one of the stages along two consecutive evolution periods. Section 3 is devoted to developing control strategies to act eventually on the larvae and adult populations, under eventual use of feedback, in order to reduce their numbers. The birth rate and/or the maximum progression rate sequences are controlled with the eventual use of feedback information to reduce the numbers of those two major stages of the evolution model. A worked illustrative example about controls implementation is also given. Section 4 presents some simulated examples to check and discuss the main theoretical results, at the levels of positivity, boundedness, and equilibrium solution, as well as 
the study of existence oscillatory solutions. Furthermore, the influences of some population control strategies on the solution sequence evolution were tested. Finally, conclusions end the paper. The main mathematical proofs are allocated in Appendix A to facilitate the reading of the main body sections.

\section{The Proposed Model, Its Basic Properties, and Worked Examples}

This section describes the basic properties of the evolution sequences of the larvae and adult subpopulations. In particular, the following topics are focused on: (a) the non-negativity of the solution sequences under non-negative initial conditions; (b) the boundedness, stability, and possible convergence to an equilibrium point; (c) the oscillatory behavior; and (d) the separate descriptions of both evolution population sequences of larvae and adult, by embedding the mutual couplings in each single stages through an extended Beverton-Holt equation.

\subsection{The Model and Its Well-Posedness}

Many of the mosquito evolution stages models assume homogeneity without separating the various metamorphic stages of mosquitoes, especially, those of the larvae and adult population stages. See, for instance, [22,23] and some of the references therein. One considers the subsequent stage-structured model of two metamorphic states, namely, larvae $x_{n}$ and adult $y_{n}$ mosquitoes at the discrete-time generation $n$. It is assumed that there are no sterile mosquitoes in the studied habitat.

$$
x_{n+1}=\frac{\alpha_{n} y_{n}}{1+\eta_{1 n} x_{n}} ; y_{n+1}=\frac{\gamma_{n} x_{n}}{1+\eta_{2 n} x_{n}} ; \forall n \in \mathbf{Z}_{0+}
$$

where $\mathbf{Z}_{0+}=\mathbf{Z}_{+} \cup\{0\}$ and $\alpha_{n}$ and $\gamma_{n}$ are the birth rate and the maximum progression rate sequences, respectively, given by

$$
\alpha_{n}=\alpha_{n}\left(x_{n}, y_{n}\right)=f_{n} k_{1 n} ; \gamma_{n}=\gamma_{n}\left(x_{n}, y_{n}\right)=g_{n} k_{2 n}
$$

and define the survival probabilities of larvae and adults as $s_{1 n}=s_{1}\left(x_{n}, y_{n}\right)$ and $s_{2 n}=s_{2}\left(x_{n}, y_{n}\right)$, given by:

$$
s_{1 n}=s_{1}\left(x_{n}, y_{n}\right)=\frac{k_{1 n}}{1+\eta_{1 n}}=\frac{\alpha_{n}}{f_{n}\left(1+\eta_{1 n}\right)} ; s_{2 n}=s_{2}\left(x_{n}, y_{n}\right)=\frac{k_{2 n}}{1+\eta_{2 n}}=\frac{\gamma_{n}}{g_{n}\left(1+\eta_{2 n}\right)}
$$

whose respective maxima are given by

$$
k_{1 n}=k_{1 n}\left(x_{n}, y_{n}\right) ; k_{2 n}=k_{2 n}\left(x_{n}, y_{n}\right)
$$

and

$$
f_{n}=f\left(x_{n}, y_{n}\right) ; g_{n}=g\left(x_{n}, y_{n}\right)
$$

are the number of off-springs produced per adult and the progression rate of larvae or the adult emergency rate, and

$$
\eta_{1 n}=\eta_{1 n}\left(x_{n}, y_{n}\right) ; \eta_{2 n}=\eta_{2 n}\left(x_{n}, y_{n}\right)
$$

are density-dependent factors. Note that $k_{1 n}$ and $k_{2 n}$ are, respectively, merged into $\alpha_{n}$ and $\gamma_{n}$. Note from (1) that total extinction of the larvae in finite time occurs in two steps ahead from the extinction of the adult if the adult extinguish, since $x_{n}=0 \Rightarrow y_{n+1}=0 \Rightarrow x_{n+2}=0$. However, $x_{n+1}=\alpha_{n} y_{n} \neq 0$ if $y_{n} \neq 0$. However, the extinction of the adult mosquitoes happens one step ahead of the extinction of the larvae, as expected, since $y_{n+1}=0$ if $x_{n}=0$.

Remark 1. Note from (2) that $\alpha_{n}$ can be reduced by external controls on either $f_{n}$ or $k_{1 n}$, or on both, by reducing, respectively, the off-springs or the survival probability of the larvae, for instance, by spreading aquatic insecticide in lagoons and unsuitable static waters, by drying unnecessary or unsuitable artificial or natural water reservoirs, 
or also by introducing in the environment sterile mosquitoes. In the same way, $\gamma_{n}$ can be reduced by external controls on either $g_{n}$ or $k_{2 n}$, or on both, by reducing, respectively, the survival probability of the adult mosquitoes, for instance, by spreading aerial insecticide in urban drains and bush water lands or by drying unsuitable artificial or natural water depots.

It is assumed through the paper that the sequences $\left\{\alpha_{n}\right\}_{n=0}^{\infty},\left\{\gamma_{n}\right\}_{n=0}^{\infty},\left\{\eta_{1 n}\right\}_{n=0}^{\infty}$, and $\left\{\eta_{2 n}\right\}_{n=0}^{\infty}$ are positive and bounded. Note that it is also possible to combine and rearrange the two above equations under the form of Beverton-Holt evolution population structures of the larvae and adult growing rules, while defining "ad hoc" carrying capacity-like and intrinsic growth rate-like parameterizations. The addendum "like" used reflects the feature that the carrying capacity and intrinsic growth rate are artificially introduced here, while having the purpose of describing the evolution of the species as it were related to a single stage, that is, a self-evolution description of either the larvae or the adult population stages. So, with such an equivalent description, the larvae and adult evolution sequences are self-generated by embedding one population stage in the other one. In this way, the mutual couplings are deleted at the expense of joining two evolution steps in each evolution equation. The information of the other species' evolution stage in each case is contained in a carrying capacity-like parameter, which depends on two previous stages, as expected. The larvae sequence self-evolution rule is as follows:

$$
x_{n+1}=\frac{\mu K_{n} x_{n}}{K_{n}+(\mu-1) x_{n}}=\frac{\alpha_{n} \gamma_{n-1} x_{n-1}}{\left(1+\eta_{1 n} x_{n}\right)\left(1+\eta_{2, n-1} x_{n-1}\right)} ; \forall n \in \mathbf{Z}_{+} .
$$

Note from the above equation that

$$
\left(1+\eta_{1 n} x_{n}\right)\left(1+\eta_{2, n-1} x_{n-1}\right) \mu K_{n} x_{n}-\left(K_{n}+(\mu-1) x_{n}\right) \alpha_{n} \gamma_{n-1} x_{n-1}=0
$$

so that,

$$
\left[\left(1+\eta_{1 n} x_{n}\right)\left(1+\eta_{2, n-1} x_{n-1}\right) \mu x_{n}-\alpha_{n} \gamma_{n-1} x_{n-1}\right] K_{n}-(\mu-1) \alpha_{n} \gamma_{n-1} x_{n} x_{n-1}=0 .
$$

Thus, the carrying capacity-like parameter of the larvae evolution is defined as follows for any intrinsic growth rate-like parameter:

$$
K_{n}=K_{n}\left(\mu, x_{n}, x_{n-1}\right)=\frac{(\mu-1) \alpha_{n} \gamma_{n-1} x_{n} x_{n-1}}{\left(1+\eta_{1 n} x_{n}\right)\left(1+\eta_{2, n-1} x_{n-1}\right) \mu x_{n}-\alpha_{n} \gamma_{n-1} x_{n-1}} ; \forall n(\geq 2) \in \mathbf{Z}_{+}
$$

with $x_{-1}=0, x_{0}>0$, and $x_{1}=\frac{\alpha_{0} x_{0}}{1+\eta_{10} x_{0}}$. Note that, as expected, this environment's carrying capacity-like parameter for the larvae evolution depends on the current and previous stages adult populations. On the other hand, one has for the adult sequence a self-evolution rule after combining both equations (1):

$$
y_{n+1}=\frac{v M_{n} y_{n}}{M_{n}+(v-1) y_{n}}=\frac{\gamma_{n} \alpha_{n-1} y_{n-1}}{1+\eta_{1, n-1} x_{n-1}+\eta_{2 n} \alpha_{n-1} y_{n-1}} .
$$

Thus, the environment carrying capacity-like parameter of the adult evolution is defined as follows from (9), and the use of its second identity under the equivalent form $x_{n-1}=\frac{y_{n}}{\gamma_{n-1}-\eta_{2, n-1} y_{n}}$ :

$$
\begin{aligned}
M_{n} & =M_{n}\left(v, y_{n}, y_{n-1}\right)=\frac{(v-1) \gamma_{n} \alpha_{n-1} y_{n} y_{n-1}}{\left(1+\eta_{1, n-1} x_{n-1}+\eta_{2 n} \alpha_{n-1} y_{n-1}\right) v y_{n}-\gamma_{n} \alpha_{n-1} y_{n-1}} \\
& =\frac{(v-1) \gamma_{n} \alpha_{n-1} y_{n} y_{n-1}\left(\gamma_{n-1}-\eta_{2, n-1} y_{n}\right)}{\left(\gamma_{n-1}-\eta_{2, n-1} y_{n}\right)\left[\left(1+\eta_{2 n} \alpha_{n-1} y_{n-1}\right) v y_{n}-\gamma_{n} \alpha_{n-1} y_{n-1}\right]+\eta_{1, n-1} y_{n} v y_{n}} .
\end{aligned}
$$

The following result stands for the non-negativity of the carrying capacity-like parameter of the larvae sequence, so as to endow it with a physical and biological sense: 
Proposition 1. $\left\{K_{n}\right\}_{n=0}^{\infty}$ Equation (8) is a non-negative real sequence if and only if

$$
\frac{x_{n}}{x_{n-1}} \geq \frac{\alpha_{n} \gamma_{n-1}}{\left(1+\eta_{1 n} x_{n}\right)\left(1+\eta_{2, n-1} x_{n-1}\right) \mu}
$$

for all $n \in Z_{+}$. If the intrinsic growth rate-like sequence is redefined in (10) as $K_{n}=K_{n}\left(\mu_{n}, x_{n}, x_{n-1}\right)$ via an intrinsic growth rate-like sequence $\left\{\mu_{n}\right\}_{n=0}^{\infty}$ by replacing $\mu \rightarrow \mu_{n}$, then the result still holds if

$$
\mu_{n} \geq \max \left(1, \frac{\alpha_{n} \gamma_{n-1} x_{n-1}}{\left(1+\eta_{1 n} x_{n}\right)\left(1+\eta_{2, n-1} x_{n-1}\right) x_{n}}\right)
$$

for all $n \in Z_{+}$.

The proof of Proposition 1 is given in Appendix A.

The subsequent result stands for the boundedness, non-negativity, and eventual extinction of the adult mosquitoes and the conditions to get them:

Proposition 2. The following properties hold:

(i) Assume that $\mu \geq 1$ and $x_{0} \geq 0$ are finite. Then, $\left\{x_{n}\right\}_{n=0}^{\infty}$ is bounded with $x_{n+1} \leq x_{n} ; \forall n \in Z_{+}$ if and only if the sequence $\left\{K_{n}\right\}_{n=0}^{\infty}$ is such that $\left\{x_{n}-K_{n}\right\}_{n=0}^{\infty}$ is non-negative, which is equivalent to $\alpha_{n} \gamma_{n-1} \geq\left(1+\eta_{1 n} x_{n}\right)\left(1+\eta_{2, n-1} x_{n-1}\right) x_{n} / x_{n-1} ; \forall n \in Z_{+}$. A necessary condition for that is that $\alpha_{n} \gamma_{n-1} \leq\left(1+\eta_{1 n} x_{n}\right)\left(1+\eta_{2, n-1} x_{n-1}\right) ; \forall n \in Z_{+}$.

In addition, $x_{n+1}<x_{n} \forall n \in Z_{+}$if $\left\{x_{n}-K_{n}\right\}_{n=0}^{N}$ is positive for any $N \in Z_{+}$and $\left\{K_{n}\right\}_{n=0}^{N} \rightarrow 0$, implying that $\left\{x_{n}-K_{n}\right\}_{n=0}^{N} \rightarrow 0$, which is equivalent to $\alpha_{n} \gamma_{n-1}>\left(1+\eta_{1 n} x_{n}\right)\left(1+\eta_{2, n-1} x_{n-1}\right) x_{n} / x_{n-1} ; \forall n \in Z_{+}$ and $\lim _{n \rightarrow \infty}\left(\alpha_{n} \gamma_{n-1} x_{n-1}-\left(1+\eta_{1 n} x_{n}\right)\left(1+\eta_{2, n-1} x_{n-1}\right) x_{n}\right)=0$.

The above results keep valid if $\mu$ is stage-dependent with $\mu_{n} \geq 1$ for all $n \in Z_{+}$.

The sequence $\left\{x_{n}\right\}_{n=0}^{\infty}$ is strictly decreasing if and only if $\left\{x_{n}-K_{n}\right\}_{n=0}^{\infty}$ is positive, that is, if and only if $\alpha_{n} \gamma_{n-1}<\left(1+\eta_{1 n} x_{n}\right)\left(1+\eta_{2, n-1} x_{n-1}\right) x_{n} / x_{n-1} ; \forall n \in Z_{+}$. A necessary condition for that is $\alpha_{n} \gamma_{n-1}<\left(1+\eta_{1 n} x_{n}\right)\left(1+\eta_{2, n-1} x_{n-1}\right) ; \forall n \in \mathbf{Z}_{+}$.

(ii) Assume that $\mu>1$ and $x_{0}>0$ are finite. Then,

$$
x_{n+1}^{-1}-\mu^{-(n+1)} \sum_{i=2}^{n} \mu^{i} \frac{\left(1+\eta_{1 i} x_{i}\right)\left(1+\eta_{2, i-1} x_{i-1}\right) \mu x_{i}-\alpha_{n} \gamma_{i-1} x_{i-1}}{\alpha_{i} \gamma_{i-1} x_{i} x_{i-1}}=x_{0} \mu^{-(n+1)}<x_{0}<+\infty ; \forall n(\geq 2) \in \mathbf{Z}_{+}
$$

and

$$
\lim _{n \rightarrow \infty}\left(x_{n+1}^{-1}-\mu^{-(n+1)} \sum_{i=2}^{n} \mu^{i} \frac{\left(1+\eta_{1 i} x_{i}\right)\left(1+\eta_{2, i-1} x_{i-1}\right) \mu x_{i}-\alpha_{n} \gamma_{i-1} x_{i-1}}{\alpha_{i} \gamma_{i-1} x_{i} x_{i-1}}\right)=x_{0} \lim _{n \rightarrow \infty} \mu^{-(n+1)}=0 .
$$

The proof of Proposition 2 is given in Appendix A.

The idea used in the proof of Proposition 2 (ii) consisted of using the population's inverses to build the evolution of the population's inverse sequence was proposed by Stevic [6] when proving the so-called Cushing-Henson conjecture with a new short proof. Basically, note that although the Beverton-Holt equation is nonlinear, its inverse is linear, which facilitates the calculation of the population evolution. The idea was later on exploited in [7-9] to introduce control theory tools for such an equation. Basically, the carrying capacity inverse can sometimes be modified by convenience in a control context by using feedback to improve the expected economic results in fisheries, aquaculture exploitations, etc. by acting, for instance, on the water temperature or food delivery in a convenient way. Further results which relate the carrying capacity sequence related to the larvae population evolution as well as solution boundedness properties are discussed in the next result. 
Proposition 3. Assume that $\left\{x_{n}-K_{n}\right\}_{n=0}^{\infty} \subseteq[0, \infty)$, for which sufficient conditions have been given in Proposition 2, and that $\mu \geq 2$. Then, $\left\{K_{n}\right\}_{n=0}^{\infty}$ is a nonnegative real sequence and $\left\{x_{n}\right\}_{n=0}^{\infty}$ is bounded if its initial value is non-negative and finite. If $\mu>2$ then $\left\{K_{n}\right\}_{n=0}^{\infty}$ is a positive sequence.

The proof of Proposition 3 is given in Appendix A. Note that Proposition 3 basically establishes that the larvae numbers at each evaluation are non-less than the carrying capacity-like parameter, as it can be expected from the parallel results in usual models based on of the Beverton-Holt equation. See, for instance, [6-11] and some references therein.

\subsection{Stability and Convergence of the Solution}

The next result proves the obvious expected fact due to the nature of the problem, that the sequences of populations of larvae and adults jointly extinguish at the same rate in the event that extinction occurs. First note from (1) that

$$
\frac{x_{n+2}}{y_{n+2}}=\frac{\alpha_{n+1}\left(1+\eta_{2, n+1} x_{n+1}\right)}{\gamma_{n+1}\left(1+\eta_{1, n+1} x_{n+1}\right)} \frac{y_{n+1}}{x_{n+1}}=\frac{\alpha_{n+1} \gamma_{n}\left(1+\eta_{2, n+1} x_{n+1}\right)\left(1+\eta_{1 n+1} x_{n}\right)}{\gamma_{n+1} \alpha_{n}\left(1+\eta_{1, n+1} x_{n+1}\right)\left(1+\eta_{2 n} x_{n}\right)} \frac{x_{n}}{y_{n}}
$$

which is being used as an auxiliary equation in the proof of the subsequent result. Such a result establishes that the populations of larvae and adults are bounded if they converge asymptotically to zero (extinction). In the event that extinction occurs, it has to jointly happen for both populations of larvae and adult, as expected by heuristic considerations.

Proposition 4. If $\left\{\frac{x_{2 n}}{y_{2 n}}\right\}_{n=0}^{\infty} \rightarrow 0$ then $\left\{\frac{y_{2 n+1}}{x_{2 n+1}}\right\}_{n=0}^{\infty} \rightarrow 0$, or if $\left\{\frac{x_{2 n}}{y_{2 n}}\right\}_{n=0}^{\infty} \rightarrow \infty$ then $\left\{\frac{y_{2 n+1}}{x_{2 n+1}}\right\}_{n=0}^{\infty} \rightarrow \infty$, it follows that $\left\{x_{n}\right\}_{n=0}^{\infty} \rightarrow 0$ and $\left\{y_{n}\right\}_{n=0}^{\infty} \rightarrow 0$, while they are also bounded as a result.

The proof of Proposition 4 is given in Appendix A.

The subsequent result establishes that, under nonnegative finite initial conditions, the sequences generated by the two equations of (1) for larvae and adult, respectively, are bounded without invoking related properties on the alternative description of the carrying capacity-like sequence, as it was done in Proposition 2.

Proposition 5. Assume that $x_{0} \geq 0$ and $y_{0} \geq 0$ are finite. Then, $\left\{x_{n}\right\}_{n=0}^{\infty}$ and $\left\{y_{n}\right\}_{n=0}^{\infty}$ are non-negative and bounded. In particular, $x_{n+1} \leq \frac{\alpha_{n} \gamma_{n-1}}{\eta_{2, n-1}}$ and $y_{n+1} \leq \frac{\gamma_{n}}{\eta_{2 n}} ; \forall n \in \mathbf{Z}_{0+}$.

The proof of Proposition 5 is given in Appendix A.

The subsequent technical result is supported by some calculations of Appendix A and it is of interest to obtain further results later on. In particular, the results emphasize both self-evolution descriptions of the larvae sequence progress and its evolution via couplings to the adult stage evolution.

Proposition 6. The following result stands to rewrite both equation (1) with equivalent expressions depending only on one of the larvae or the adult populations.

$$
\begin{gathered}
x_{n}=\frac{y_{n+1}}{\gamma_{n}-\eta_{2 n} y_{n+1}} \text { for } n \geq 1 \\
x_{n+1}=\frac{\left(\gamma_{n}-\eta_{2 n} y_{n+1}\right) \alpha_{n} y_{n}}{\gamma_{n}+\left(\eta_{1 n}-\eta_{2 n}\right) y_{n+1}} \text { for } n \geq 1 \\
x_{n+1}=\frac{\alpha_{n} \gamma_{n-1} x_{n-1}}{\left(1+\eta_{1 n} x_{n}\right)\left(1+\eta_{2, n-1} x_{n-1}\right)} \text { for } n \geq 1
\end{gathered}
$$




$$
x_{n+1}=\frac{\left(\gamma_{n}-\eta_{2 n} y_{n+1}\right) \alpha_{n} y_{n}\left[\gamma_{n-1}+\left(\eta_{1, n-1}-\eta_{2, n-1}\left(1+\eta_{2 n} \alpha_{n-1} y_{n-1}\right)\right) y_{n}+\eta_{2 n} \gamma_{n-1} \alpha_{n-1} y_{n-1}\right]}{\gamma_{n}\left[\gamma_{n-1}+\left(\eta_{1, n-1}-\eta_{2, n-1}\left(1+\eta_{2 n} \alpha_{n-1} y_{n-1}\right)\right) y_{n}+\eta_{2 n} \gamma_{n-1} \alpha_{n-1} y_{n-1}\right]+\left(\eta_{1 n}-\eta_{2 n}\right) \gamma_{n}\left(\gamma_{n-1}-\eta_{2, n-1} y_{n}\right) \alpha_{n-1} y_{n-1}}
$$

The proof of Proposition 6 is given in Appendix A.

The subsequent result discusses the existence and stability and the nonexistence of a positive equilibrium point. See also [1] and [12] for similar equilibrium studies under invariant parameterizations in this kind of model and $[13,14]$ for studies of convergence of classes of more general discrete sequences to their equilibrium points.

Proposition 7. Assume that $\left\{\alpha_{n}\right\}_{n=0}^{\infty} \rightarrow \alpha,\left\{\gamma_{n}\right\}_{n=0}^{\infty} \rightarrow \gamma,\left\{\eta_{1 n}\right\}_{n=0}^{\infty} \rightarrow \eta_{1}$, and $\left\{\eta_{2 n}\right\}_{n=0}^{\infty} \rightarrow \eta_{2}$ with those limits being positive. Then, the following properties hold:

There exists a unique globally stable non-negative equilibrium point $\left(x_{e}, y_{e}\right)$ of (1), if and only if $\alpha \gamma \geq 1$ implying extinction, that is $\left(x_{e}, y_{e}\right)=(0,0)$, if and only if $\alpha \gamma=1$. The nonzero equilibrium point is

$$
\begin{gathered}
x_{e 1}=\frac{\sqrt{\left(\eta_{1}+\eta_{2}\right)^{2}+4 \eta_{1} \eta_{2}(\alpha \gamma-1)}-\left(\eta_{1}+\eta_{2}\right)}{2 \eta_{1} \eta_{2}} ; \\
y_{e}=\frac{\gamma\left(\sqrt{\left(\eta_{1}+\eta_{2}\right)^{2}+4 \eta_{1} \eta_{2}(\alpha \gamma-1)}-\eta_{1}-\eta_{2}\right)}{2 \eta_{1} \eta_{2}+\eta_{2}\left(\sqrt{\left(\eta_{1}+\eta_{2}\right)^{2}+4 \eta_{1} \eta_{2}(\alpha \gamma-1)}-\eta_{1}-\eta_{2}\right)} .
\end{gathered}
$$

If the equilibrium point is zero then it is not locally asymptotically stable.

The positive equilibrium point is locally asymptotically stable if and only if $1<\sqrt{\alpha \gamma}<\frac{1}{2}\left(1-\frac{\eta_{2}}{\eta_{1}}\right)+$ $\frac{\sqrt{\left(\eta_{1}+\eta_{2}\right)^{2}+4 \eta_{1} \eta_{2}(\alpha \gamma-1)}}{2 \eta_{1}}$.

A sufficient condition for Property (iii) to hold is $\frac{1}{2}\left(1-\frac{\eta_{2}}{\eta_{1}}\right)>\sqrt{\alpha \gamma}-\frac{\sqrt{\left(\eta_{1}+\eta_{2}\right)^{2}+4 \eta_{1} \eta_{2}(\alpha \gamma-1)}}{2 \eta_{1}}$ and a necessary condition for such a condition to hold is

$$
1>\sqrt{\alpha \gamma}-\frac{\sqrt{\eta_{1} \eta_{2}} \sqrt{\alpha \gamma-1}}{\eta_{1}}
$$

The proof of Proposition 7 is given in Appendix A.

Proposition 7 is now revisited under the removal of the assumption $\left\{\alpha_{n}\right\}_{n=0}^{\infty} \rightarrow \alpha,\left\{\gamma_{n}\right\}_{n=0}^{\infty} \rightarrow \gamma$, $\left\{\eta_{1 n}\right\}_{n=0}^{\infty} \rightarrow \eta_{1}$, and $\left\{\eta_{2 n}\right\}_{n=0}^{\infty} \rightarrow \eta_{2}$.

Proposition 8. Assume that

(1) $\frac{1}{\gamma_{n}} \leq \alpha_{n}<\frac{\alpha_{n-1} \gamma_{n-2} x_{n-2}\left(1+\eta_{1 n} x_{n}\right)\left(1+\eta_{2, n-1} x_{n-1}\right)}{\gamma_{n-1} x_{n-1}\left(1+\eta_{1, n-1} x_{n-1}\right)\left(1+\eta_{2, n-2} x_{n-2}\right)} \leq 0 ; \forall n\left(\geq n_{0}\right) \in \mathbf{Z}_{0+}$ for some $n_{0} \in \mathbf{Z}_{0+}$ ，

(2) $\limsup _{n \rightarrow \infty}\left(\alpha_{n}-\frac{\alpha_{n-1} \gamma_{n-2} x_{n-2}\left(1+\eta_{1 n} x_{n}\right)\left(1+\eta_{2, n-1} x_{n-1}\right)}{\gamma_{n-1} x_{n-1}\left(1+\eta_{1, n-1} x_{n-1}\right)\left(1+\eta_{2, n-2} x_{n-2}\right)}\right) \leq 0$,

(3) $\liminf _{n \rightarrow \infty}\left(\alpha_{n}-\gamma_{n}^{-1}\right) \geq 0$.

Then, the populations of larvae and adult mosquitoes converge asymptotically to zero at an exponential rate. The result folds if condition 2 is replaced with the stronger one:

$$
\limsup _{n \rightarrow \infty}\left(\alpha_{n}-\frac{\alpha_{n-1} \gamma_{n-2} x_{n-2}\left(1+\eta_{1 n} x_{n}\right)\left(1+\eta_{2, n-1} x_{n-1}\right)}{\gamma_{n-1} x_{n-1}\left(1+\eta_{1, n-1} x_{n-1}\right)\left(1+\eta_{2, n-2} x_{n-2}\right)}\right) \leq 0 .
$$

The proof of Proposition 8 is given in Appendix A. 
Since the sequences $\left\{x_{n}\right\}_{n=0}^{\infty}$ and $\left\{y_{n}\right\}_{n=0}^{\infty}$ are non-negative and bounded, then there exist non-negative real constants $X_{m}, X_{M}\left(\geq X_{m}\right), Y_{m}$, and $Y_{M}\left(\geq Y_{m}\right)$, such that $X_{m} \leq x_{n} \leq X_{M}$ and $Y_{m} \leq y_{n} \leq Y_{M} ; \forall n \in Z_{0+}$. Those inequalities exhibit boundedness and stability of the populations of larvae and adults, and are also compatible with their potential convergence to the equilibrium points and with eventual presence of bounded oscillations discussed later on. Note also that the constants $X_{m}, X_{M}, Y_{m}$ and are related as follows:

$$
\begin{gathered}
X_{m} \leq x_{n+1}=\frac{\alpha_{n} y_{n}}{1+\eta_{1 n} x_{n}} \leq \frac{\alpha_{n} Y_{M}}{1+\eta_{1 n} X_{m}} \Rightarrow Y_{M} \geq \frac{X_{m}}{\alpha_{n}}\left(1+\eta_{1 n} X_{m}\right) \\
X_{M} \geq x_{n+1}=\frac{\alpha_{n} y_{n}}{1+\eta_{1 n} x_{n}} \geq \frac{\alpha_{n} Y_{m}}{1+\eta_{1 n} X_{M}} \Rightarrow Y_{m} \leq \frac{X_{M}}{\alpha_{n}}\left(1+\eta_{1 n} X_{M}\right)
\end{gathered}
$$

Again note from (1) that

$$
x_{n+1}=\frac{\alpha_{n} \gamma_{n-1} x_{n-1}}{\left(1+\eta_{1 n} x_{n}\right)\left(1+\eta_{2, n-1} x_{n-1}\right)} \leq \min \left(\frac{\alpha_{n} Y_{M}}{1+\eta_{1 n} X_{m}}, \frac{\alpha_{n} \gamma_{n-1} X_{M}}{\left(1+\eta_{1 n} X_{m}\right)\left(1+\eta_{2, n-1} X_{m}\right)}\right) .
$$

If $g_{n}=x_{n+1} / x_{n}$, one gets:

$$
g_{n} \leq g_{0 n M}=\frac{\alpha_{n}}{X_{m}\left(1+\eta_{1 n} X_{m}\right)} \min \left(Y_{M}, \frac{\gamma_{n-1} X_{M}}{1+\eta_{2, n-1} X_{m}}\right)
$$

A refinement of the above bound can be obtained by writing $x_{n}=g_{n-1} x_{n-1}$ leading to

$$
g_{n} \leq g_{1 n M}=\frac{\alpha_{n}}{X_{m}\left(1+\eta_{1 n} g_{n-1} X_{m}\right)} \min \left(Y_{M}, \frac{\gamma_{n-1} X_{M}}{1+\eta_{2, n-1} X_{m}}\right) .
$$

The combined use of the two above upper bounds leads to

$$
g_{n} \leq g_{n M}=\frac{\alpha_{n}}{X_{m}} \min \left(Y_{M}, \frac{\gamma_{n-1} X_{M}}{1+\eta_{2, n-1} X_{m}}\right) \min \left[\frac{1}{1+\eta_{1 n} X_{m}}, \frac{1}{1+\eta_{1 n} g_{n-1} X_{m}}\right]
$$

Also, one can get lower bounds as follows:

$$
\begin{gathered}
g_{n} \geq g_{0 n m}=\frac{\alpha_{n}}{X_{M}\left(1+\eta_{1 n} X_{M}\right)} \max \left(Y_{m}, \frac{\gamma_{n-1} X_{m}}{1+\eta_{2, n-1} X_{M}}\right) \\
g_{n} \geq g_{1 n m}=\frac{\alpha_{n}}{X_{M}\left(1+\eta_{1 n} g_{n-1} X_{M}\right)} \max \left(Y_{m}, \frac{\gamma_{n-1} X_{m}}{1+\eta_{2, n-1} X_{M}}\right) \\
g_{n} \geq g_{n m}=\frac{\alpha_{n}}{X_{M}} \max \left(Y_{m}, \frac{\gamma_{n-1} X_{m}}{1+\eta_{2, n-1} X_{M}}\right) \max \left[\frac{1}{1+\eta_{1 n} X_{M}}, \frac{1}{1+\eta_{1 n} g_{n-1} X_{M}}\right] .
\end{gathered}
$$

Note also that we can use the expression $x_{n}=\left[\prod_{i=k}^{n-1} g_{i}\right] x_{k}$ for any $k \in Z_{0+}$. This yields the sets of upper bounds and lower bounds:

$$
\begin{aligned}
& g_{n} \leq g_{n M}(k, n)=\frac{\alpha_{n}}{X_{m}} \min \left(Y_{M}, \frac{\gamma_{n-1} X_{M}}{1+\eta_{2, n-1}\left[\prod_{i=k}^{n-2} g_{i}\right] X_{m}}\right) \min \left[\frac{1}{1+\eta_{1 n} X_{m}}, \frac{1}{1+\eta_{1 n}\left[\prod_{i=k}^{n-1} g_{i}\right] X_{m}}\right] ; \forall k \in Z_{0+} \\
& g_{n} \geq g_{n m}(k, n)=\frac{\alpha_{n}}{X_{M}} \max \left(Y_{m}, \frac{\gamma_{n-1} X_{m}}{1+\eta_{2, n-1}\left[\prod_{i=k}^{n-2} g_{i}\right] X_{M}}\right) \max \left[\frac{1}{1+\eta_{1 n} X_{M}}, \frac{1}{1+\eta_{1 n}\left[\prod_{i=k}^{n-2} g_{i}\right] X_{M}}\right] ; \forall k \in Z_{0+}
\end{aligned}
$$

In the following result, one considers potential constant sequences as the trivial case of nonstrict oscillatory sequences in the most general case. For other interesting investigations of oscillatory solutions in discrete sequences, one can refer to $[10,12]$

Proposition 9. The subsequent properties hold: 
(i) Assume that for each given $n \in \mathbf{Z}_{0+}$, there exist finite $k_{1}=k_{1}(n) \in \mathbf{Z}_{0+}$ and $k_{2}=k_{2}\left(k_{1}(n)\right)=$ $k_{2}(n)\left(>k_{1}\right) \in Z_{+}$, such that either $\prod_{i=n}^{n+k_{1}(n)}\left[g_{i}\right] \leq 1$ and $\prod_{i=n+k_{1}(n)+1}^{n+k_{2}(n)}\left[g_{i}\right] \geq 1$ or $\prod_{i=n}^{n+k_{1}(n)}\left[g_{i}\right] \geq 1$ and $\prod_{i=n+k_{1}(n)+1}^{n+k_{2}(n)}\left[g_{i}\right] \leq 1$. Then, the mosquito larvae evolution solution $\left\{x_{n}\right\}_{n=0}^{\infty}$ is oscillatory.

The solution $\left\{x_{n}\right\}_{n=0}^{\infty}$ is strictly oscillatory if in each of the above double conditions at least one of the inequalities is strict.

The oscillatory solution $\left\{x_{n}\right\}_{n=0}^{\infty}$ is periodic of, in general, a time-varying (or stage-dependent) oscillation period, if and only if there exists a strictly increasing sequence $\left\{k_{n}\right\}_{n=0}^{\infty}$ with $k_{n+1}-k_{n} \leq k_{s}<+\infty$, such that $\prod_{i=k_{j}}^{k_{j+1}}\left[g_{i}\right]=1 ; \forall j \in \mathbf{Z}_{0+}$.

(ii) A sufficient condition for the solution $\left\{x_{n}\right\}_{n=0}^{\infty}$ to be oscillatory is that for any fixed non-negative integer number or any set of non-negative integer numbers (depending on the available computational possibilities) $k(i) \leq i-2$ for $i=n, n+1, \ldots, n+k_{2}$ and each $n \in \mathbf{Z}_{0+}$ :

$$
\begin{aligned}
& \text { either } \prod_{i=n}^{n+k_{1}(n)}\left[g_{i M}(k(i), i)\right] \leq 1 \text { and } \prod_{i=n+k_{1}(n)+1}^{n+k_{2}(n)}\left[g_{i m}(k(i), i)\right] \leq 1, \\
& \quad \text { or } \prod_{i=n}^{n+k_{1}(n)}\left[g_{i m}(k(i), i)\right] \geq 1 \text { and } \prod_{i=n+k_{1}(n)+1}^{n+k_{2}(n)}\left[g_{i M}(k(i), i)\right] \leq 1 .
\end{aligned}
$$

(iii) Different alternative sufficiency-type conditions for the solution $\left\{x_{n}\right\}_{n=0}^{\infty}$ to be oscillatory are obtained with any of the following replacements in the sufficiency-type conditions of Property (ii):

$g_{i M}(k(i), i) \rightarrow g_{0 i M}, g_{1 i M}, g_{2 i M}$ or by the alternative use of any of the combination of minima appearing in their definitions,

$g_{i m}(k(i), i) \rightarrow g_{0 i m}, g_{1 i m}, g_{2 i m}$ or by the alternative use of any of the combination of maxima appearing in their definitions.

It turns out that an oscillatory solution of the larvae solution sequence does not necessarily imply an oscillatory solution of the adult sequence.

\subsection{Worked Examples}

Example 1. Oscillatory larvae solution of period two. From (1), one can write equivalently:

$$
y_{n}=\left(1+\eta_{1 n} x_{n}\right) \frac{x_{n+1}}{\alpha_{n}} ; y_{n+1}=\frac{\gamma_{n} x_{n}}{1+\eta_{2 n} x_{n}}
$$

so that

$$
\frac{y_{n+1}}{y_{n}}=\frac{\alpha_{n} \gamma_{n} x_{n}}{\left(1+\eta_{1 n} x_{n}\right)\left(1+\eta_{2 n} x_{n}\right) x_{n+1}}=\frac{\alpha_{n} \gamma_{n}}{\left(1+\eta_{1 n} x_{n}\right)\left(1+\eta_{2 n} x_{n}\right) g_{n}}
$$

then

$$
\frac{\alpha_{n} \gamma_{n}}{\left(1+\eta_{1 n} X_{M}\right)\left(1+\eta_{2 n} X_{M}\right)} \max \left(\frac{X_{m}}{X_{M}}, \frac{1}{g_{n}}\right) \leq \frac{y_{n+1}}{y_{n}} \leq \frac{\alpha_{n} \gamma_{n}}{\left(1+\eta_{1 n} X_{m}\right)\left(1+\eta_{2 n} X_{m}\right)} \min \left(\frac{X_{M}}{X_{m}}, \frac{1}{g_{n}}\right)
$$

so that if $x_{n+1}=g_{n} x_{n}$ and $y_{n+1}=f_{n} y_{n}$ then:

(a) If $g_{n} \geq 1$ then $\frac{\alpha_{n} \gamma_{n}}{\left(1+\eta_{1 n} X_{M}\right)\left(1+\eta_{2 n} X_{M}\right)} \max \left(\frac{X_{m}}{X_{M}}, \frac{1}{g_{n}}\right) \leq f_{n} \leq \frac{\alpha_{n} \gamma_{n}}{\left(1+\eta_{1 n} X_{m}\right)\left(1+\eta_{2 n} X_{m}\right) g_{n}}$.

(b) If $g_{n} \leq 1$ then $\frac{\alpha_{n} \gamma_{n}}{\left(1+\eta_{1 n} X_{M}\right)\left(1+\eta_{2 n} X_{M}\right) g_{n}} \leq f_{n} \leq \frac{\alpha_{n} \gamma_{n}}{\left(1+\eta_{1 n} X_{m}\right)\left(1+\eta_{2 n} X_{m}\right)} \min \left(\frac{X_{M}}{X_{m}}, \frac{1}{g_{n}}\right)$.

Note that an oscillation in the larvae solution sequence does not necessarily imply an oscillation in the adult solution sequence of the same period. 
Assume for instance that $g_{2 n}>1$ and $g_{2 n+1}<1$ for any $n \in \mathbf{Z}_{0+}$. It is possible that

$$
f_{2 n} \leq \frac{\alpha_{2 n} \gamma_{2 n}}{\left(1+\eta_{1,2 n} X_{m}\right)\left(1+\eta_{2,2 n} X_{m}\right) g_{2 n}} \leq 1 \text { with }_{2 n} \geq \frac{\alpha_{2 n} \gamma_{2 n}}{\left(1+\eta_{1,2 n} X_{m}\right)\left(1+\eta_{2,2 n} X_{m}\right)}>1 ; \forall n \in Z_{0+}
$$

if $\alpha_{2 n} \gamma_{2 n}>\left(1+\eta_{1,2 n} X_{m}\right)\left(1+\eta_{2,2 n} X_{m}\right) ; \forall n \in Z_{0+}$, and $f_{2 n+1} \leq \frac{\alpha_{2 n+1} \gamma_{2 n+1}}{\left(1+\eta_{1,2 n+1} X_{m}\right)\left(1+\eta_{2,2 n+1} X_{m}\right) g_{2 n+1}} \leq 1$, with $1>g_{2 n+1} \geq \frac{\alpha_{2 n+1} \gamma_{2 n+1}}{\left(1+\eta_{1,2 n+1} X_{m}\right)\left(1+\eta_{2,2 n+1} X_{m}\right)} ; \forall n \in Z_{0+}$.

if $\alpha_{2 n+1} \gamma_{2 n+1} \leq\left(1+\eta_{1,2 n+1} X_{m}\right)\left(1+\eta_{2,2 n+1} X_{m}\right) g_{2 n+1}<\left(1+\eta_{1,2 n+1} X_{m}\right)\left(1+\eta_{2,2 n+1} X_{m}\right) ; \forall n \in Z_{0+}$. So, in this case, the larvae solution has an oscillatory solution of period two, while the adult solution has no oscillatory solution of such a period.

Example 2. Limit cycle of two levels of the larvae solution for model time-varying parameterization. Assume that for some $n_{0} \in \mathbf{Z}_{0+}$ and any $n\left(\geq n_{0}+1\right) \in \mathbf{Z}_{+}$, one has a solution $x_{n+1}=x_{n-1}=a x_{n-2}=x_{n}=b$ for the larvae evolution then one has from Equation (A2) of Appendix A that $\frac{\alpha_{n} \gamma_{n-1}}{\left(1+\eta_{1 n} b\right)\left(1+\eta_{2, n-1} a\right)}=\frac{\alpha_{n+1} \gamma_{n}}{\left(1+\eta_{1, n+1} a\right)\left(1+\eta_{2 n} b\right)}=1$ and then

$$
\alpha_{n}=\frac{\left(1+\eta_{1 n} b\right)\left(1+\eta_{2, n-1} a\right)}{\gamma_{n-1}}, \alpha_{n+1}=\frac{\left(1+\eta_{1, n+1} a\right)\left(1+\eta_{2 n} b\right)}{\gamma_{n}} .
$$

Therefore, the birth rate subsequence

$$
\left\{\alpha_{n}\right\}_{n_{0}}^{\infty}=\left\{\frac{\left(1+\eta_{1 n} b\right)\left(1+\eta_{2, n-1} a\right)}{\gamma_{n-1}}, \frac{\left(1+\eta_{1, n+1} a\right)\left(1+\eta_{2 n} b\right)}{\gamma_{n}}, \frac{\left(1+\eta_{1 n+2} b\right)\left(1+\eta_{2 n} a\right)}{\gamma_{n+1}}, \ldots\right\}
$$

generates a cycle of a period of two stages in the larvae solution sequence on $\left[n_{0}+1, \infty\right)$, given by the subsequence $\left\{x_{n}\right\}_{n_{0}+1}^{\infty}=\{a, b, a, b, a, \ldots \ldots\} \subset\left\{x_{n}\right\}_{n=0}^{\infty}$. If $a=b$ then this subsequence of the solution is, in particular, constant. The above periodic oscillatory sequence for the larvae evolution does not imply that the solution for the adults' evolution necessarily also has a periodic solution subsequence. To this end, additional constraints are needed. Now, assume that for some $n_{01} \in Z_{0+}$ and any $n\left(\geq n_{01}+1\right) \in Z_{+}$, one has a solution $y_{n+1}=y_{n-1}=c y_{n-2}=y_{n}=d$ for the adult mosquito evolution. Then, one can conclude from (A.3) of Appendix A that

$$
x_{n+1}=\frac{\alpha_{n} y_{n}}{1+\eta_{1 n} x_{n}} ; y_{n+1}=\frac{\gamma_{n} x_{n}}{1+\eta_{2 n} x_{n}} ;
$$

and then

$$
\begin{gathered}
\gamma_{n}=\frac{\gamma_{n-1}+\left(\eta_{1, n-1}-\eta_{2, n-1}\left(1+\eta_{2 n} \alpha_{n-1} c\right)\right) d+\eta_{2 n} \gamma_{n-1} \alpha_{n-1} c}{\alpha_{n-1}\left(\gamma_{n-1}-\eta_{2, n-1} d\right)} \\
\gamma_{n+1}=\frac{\gamma_{n}+\left(\eta_{1 n}-\eta_{2 n}\left(1+\eta_{2, n+1} \alpha_{n} d\right)\right) c+\eta_{2, n+1} \gamma_{n} \alpha_{n} d}{\alpha_{n}\left(\gamma_{n}-\eta_{2 n} c\right)} .
\end{gathered}
$$

Therefore, the progression rate subsequence

$$
\left\{\gamma_{n}\right\}_{n_{01}}^{\infty}=\left\{\begin{array}{c}
\frac{\gamma_{n-1}+\left(\eta_{1, n-1}-\eta_{2, n-1}\left(1+\eta_{2 n} \alpha_{n-1} c\right)\right) d+\eta_{2 n} \gamma_{n-1} \alpha_{n-1} c}{\alpha_{n-1}\left(\gamma_{n-1}-\eta_{2, n-1} d\right)}, \frac{\gamma_{n}+\left(\eta_{1 n}-\eta_{2 n}\left(1+\eta_{2, n+1} \alpha_{n} d\right)\right) c+\eta_{2, n+1} \gamma_{n} \alpha_{n} d}{\alpha_{n}\left(\gamma_{n}-\eta_{2 n} c\right)} \\
\left., \frac{\gamma_{n+1}+\left(\eta_{1, n+1}-\eta_{2, n+1}\left(1+\eta_{2, n+2} \alpha_{n} c\right)\right) d+\eta_{2, n+2} \gamma_{n} \alpha_{n} c}{\alpha_{n+1}\left(\gamma_{n+1}-\eta_{2, n+1} d\right)}, \ldots\right\}
\end{array}\right.
$$

generates a cycle of a period of two stages in the adult solution sequence on $\left[n_{01}+1, \infty\right)$ given by the subsequence $\left\{y_{n}\right\}_{n_{01}+1}^{\infty}=\{c, d, c, d, c, \ldots \ldots\} \subset\left\{x_{n}\right\}_{n=0}^{\infty}$. Note that both cycles are achievable in an independent fashion, each through its respective sequence of gains. If the birth and the progression rates are generated by (17) and (18), then both solutions are periodic of period two stages on the interval $\max \left(n_{0}, n_{01}\right)+1$. On the other hand, if the birth rate (17) does not hold for $n \geq n_{0}$, but instead one has:

$$
\lim _{n \rightarrow \infty}\left(\alpha_{2 n+1}-\frac{\left(1+\eta_{1,2 n+1} b\right)\left(1+\eta_{2,2 n-1} a\right)}{\gamma_{2 n}}\right)=0, \lim _{n \rightarrow \infty}\left(\alpha_{2 n}-\frac{\left(1+\eta_{1,2 n} a\right)\left(1+\eta_{2,2 n-1} b\right)}{\gamma_{2 n-1}}\right)=0
$$


then, the solution of the larvae evolution $\left\{x_{n}\right\}_{n_{0}+1}^{\infty}$ converges to a limit cycle. A similar conclusion applies for the solution sequence of the adult mosquitoes if (18) does not hold, while it is replaced with a limit condition for the progression rate.

Example 3. Limit cycle of two levels of the larvae solution for model constant parameterization. If a stage-independent parameterization is considered in Example 2 with $\alpha_{n}=\alpha, \gamma_{n}=\gamma, \eta_{1 n}=\eta_{1}, \eta_{2 n}=\eta_{2}$; $\forall n \in Z_{0+}$, then one can conclude from the two equations of (1) in Example 2 that

$$
\begin{aligned}
& a=\frac{\alpha d}{1+\eta_{1} b}, b=\frac{\alpha c}{1+\eta_{1} a} \\
& c=\frac{\gamma b}{1+\eta_{2} b}, d=\frac{\gamma a}{1+\eta_{2} a}
\end{aligned}
$$

subject to $\max (c, d)<\gamma / \eta_{2}$, since $a=\frac{d}{\gamma-\eta_{2} d}$ and $b=\frac{c}{\gamma-\eta_{2} c}$, in order to guarantee the non-negativity and boundedness of the oscillation if it exists. Furthermore, direct calculations with the various equations in (19) conclude that

$$
\begin{gathered}
\frac{a}{b}=\frac{d}{c} \frac{1+\eta_{1} a}{1+\eta_{1} b}, \\
\frac{c}{d}=\frac{b}{a} \frac{1+\eta_{2} a}{1+\eta_{2} b}=\frac{c}{d} \frac{1+\eta_{1} b}{1+\eta_{1} a} \frac{1+\eta_{2} a}{1+\eta_{2} b} \Rightarrow \frac{\left(1+\eta_{1} b\right)\left(1+\eta_{2} a\right)}{\left(1+\eta_{1} a\right)\left(1+\eta_{2} b\right)}=1 \Rightarrow(a-b) \eta_{1}=(a-b) \eta_{2} .
\end{gathered}
$$

Note that:

(a) At the equilibrium point, $a=b$ so that $\eta_{1}$ and $\eta_{2}$ can be either identical or distinct.

(b) If a cycle of a two-stage period being distinct of an equilibrium point occurs, then $a \neq b$ so that $\eta=\eta_{1}=\eta_{2}$.

In the above second case, Equation (19) becomes:

$$
\begin{aligned}
& a=\frac{\alpha d}{1+\eta b}, b=\frac{\alpha c}{1+\eta a} \\
& c=\frac{\gamma b}{1+\eta b}, d=\frac{\gamma a}{1+\eta a} .
\end{aligned}
$$

Thus,

$$
\begin{aligned}
c=\frac{\gamma b}{1+\eta b}=\frac{\gamma}{1+\eta b} \frac{\alpha c}{1+\eta a} & \Rightarrow \frac{\gamma}{1+\eta b} \frac{\alpha}{1+\eta a}=1 \Rightarrow 1+\eta b=\frac{\alpha \gamma}{1+\eta a} \Rightarrow \\
b & =b(a)=\frac{\alpha \gamma-\eta a-1}{(1+\eta a) \eta}
\end{aligned}
$$

which implies in addition that for the existence of non-negative cycle values, a has to be small enough to satisfy the constraint $a<\frac{\alpha \gamma-1}{\eta}$. Also,

$$
\begin{gathered}
c=c(a)=\frac{\gamma(\alpha \gamma-\eta a-1)}{\eta(1+\eta a)(1+\eta b)} \\
d=d(a)=\frac{\gamma a}{1+\eta a} .
\end{gathered}
$$

As a result, any two-stage period cycle, for both non-negative populations of larvae and adult mosquitoes, that is not coincident with the equilibrium point, has to satisfy $\eta_{1}=\eta_{2}, \alpha \gamma>1, a<\frac{\alpha \gamma-1}{\eta}$, and for a given a, $b(a), c(a)$, and $d(a)$ are given by (22)-(24). The extinction case is obtained as a particular case, implying that the cycle coincides with the zero equilibrium point under a nonstrict upper bounding constraint of a leading to $0=a \leq \frac{\alpha \gamma-1}{\eta}$, implying $\alpha \gamma=1$ and $b=c=d=0$ from (21) and (22).

\section{Monitored Control of the Mosquito Populations}

It has been verified that the intensity of transmission can be determined through the vectorial capacity, equivalent to the basic reproduction ratio of a disease. It describes the total number of potentially infectious bites that would occur from all the mosquitoes in an area biting a single infective human along a single day. The so-called McDonald model is very sensitive to interventions focused on adult mosquitoes. In fact, such interventions cause a reduction in both the probability of vector 
survival and the ratio of vector to humans. The following items have to be properly identified and fixed for the monitored control intervention:

(a) The physical space of intervention;

(b) The intensity and periodicity of the intervention, taking into account general details and information, such as seasonality influencing temperature, density, and larval food amounts availability;

(c) The appropriate period of the day of intervention, for instance, at nights, for the case of Anopheles, where mosquitoes are more active;

(d) The use of large allowed amounts of insecticide compatible with the ecosystem preservation and limited health influence damage.

Details on potential control strategies and protocols to follow can be found in $[24,25]$ and some references therein. The proposed controls are performed by manipulation of the progression rates which depend, in general, on temperature, larval diet and density effects, and, of course, on the type of mosquito [26,27].

The use of controls, subject to feedback information, for the monitored reductions of larvae and adult mosquito populations is developed in this section. Its practical implementation has to take into account the constraints generated by the considerations of the above items a) to c) by the insecticide company's planning.

Assume that for some real sequences $\left\{\widetilde{\alpha}_{n}\right\}_{n=0}^{\infty} \subseteq[0,1]$ and $\left\{\widetilde{\gamma}_{n}\right\}_{n=0}^{\infty} \subseteq[0,1], \alpha_{n}=\left(1-\widetilde{\alpha}_{n}\right) \alpha$; $\gamma_{n}=\left(1-\widetilde{\gamma}_{n}\right) \gamma$. Then, the interpretation of the necessary and sufficient condition of Proposition 2 guarantees that $x_{n+1} \leq x_{n} ; \forall n \in Z_{+}$is if $x_{n-1} \neq 0$

$$
\begin{aligned}
\left(1-\widetilde{\alpha}_{n}\right)\left(1-\widetilde{\gamma}_{n}\right) & =1+\widetilde{\alpha}_{n} \widetilde{\gamma}_{n}-\left(\widetilde{\alpha}_{n}+\widetilde{\gamma}_{n}\right) \leq\left(1+\eta_{1 n} x_{n}\right)\left(1+\eta_{2, n-1} x_{n-1}\right) x_{n} / \alpha \gamma x_{n-1} \\
= & \left(1+\eta_{1 n} \eta_{2, n-1} x_{n} x_{n-1}+\eta_{1 n} x_{n}+\eta_{2, n-1} x_{n-1}\right) / \alpha \gamma x_{n-1} \\
\leq & \left(1+\eta_{1 n} \eta_{2, n-1} x_{n-1}^{2}+2\left(\eta_{1 n}+\eta_{2, n-1}\right) x_{n-1}\right) / \alpha \gamma x_{n-1} \\
= & \left(1+\eta_{1 n} \eta_{2, n-1} x_{n-1}+2\left(\eta_{1 n}+\eta_{2, n-1}\right)\right) / \alpha \gamma ; \forall n \in \mathbf{Z}_{+}
\end{aligned}
$$

or

$$
\widetilde{\alpha}_{n} \widetilde{\gamma}_{n}-\left(\widetilde{\alpha}_{n}+\widetilde{\gamma}_{n}\right) \leq \frac{1}{\alpha \gamma}\left(\eta_{1 n} \eta_{2, n-1} x_{n-1}+2\left(\eta_{1 n}+\eta_{2, n-1}\right)\right)+\frac{1-\alpha \gamma}{\alpha \gamma} .
$$

Since $0 \leq \alpha \gamma \leq 1$ and $0 \leq \widetilde{\alpha}_{n} \widetilde{\gamma}_{n} \leq 1$, then the above inequality is trivially true, since any nonpositive real number is less than or equal to some non-negative real number.

The density factors $\eta_{1 n}$ and $\eta_{2 n}$ could decrease as the populations decrease by the action of the controls, leading to an increase of the survival probabilities. However, this feature is neglected in the model.

Remark 2. Note from the subsequent equilibrium point equations obtained in Proposition 7:

$$
x_{e 2}=-\frac{\sqrt{\left(\eta_{1}+\eta_{2}\right)^{2}+4 \eta_{1} \eta_{2}(\alpha \gamma-1)}+\left(\eta_{1}+\eta_{2}\right)}{2 \eta_{1} \eta_{2}} ; y_{e}=\frac{\gamma x_{e}}{1+\eta_{2} x_{e}} .
$$

that the equilibrium point under the controls has a smaller value related to the control-free situation if $\alpha$ is replaced with $\alpha_{c}<\alpha$ and $\gamma$ is replaced with $\gamma_{c}<\gamma$, being smaller limits of the corresponding gain sequences, assumed to be convergent, through the use of insecticide dropping or the introduction or sterile mosquitoes, under the kept constraint $\alpha \gamma \geq 1$, compared to the control-free case.

Now, Equation (7) becomes modified as follows:

$$
x_{n+1}=\frac{\mu K_{n} x_{n}}{K_{n}+(\mu-1) x_{n}}=\frac{\alpha \gamma\left(1-\widetilde{\alpha}_{n}\right)\left(1-\widetilde{\gamma}_{n-1}\right) x_{n-1}}{\left(1+\eta_{1 n} x_{n}\right)\left(1+\eta_{2, n-1} x_{n-1}\right)} .
$$


The decreasing of the larvae population at the $(n+1)$ stage by the control action related to the uncontrolled case is $\frac{x_{n+1}}{x_{n+1}^{0}}=\left(1-\widetilde{\alpha}_{n}\right)\left(1-\widetilde{\gamma}_{n-1}\right)$. From the inverses of Equation (1), and provided that $x_{n} \neq 0, y_{n} \neq 0$, one obtains the following evolutions of the inverse populations of larvae and adults:

$$
x_{n+1}^{-1}=\frac{1+\eta_{1 n} x_{n}}{\alpha_{n} y_{n}}=\alpha_{n}^{-1} y_{n}^{-1}\left(1+\eta_{1 n} x_{n}\right) ; y_{n+1}^{-1}=\frac{1+\eta_{2 n} x_{n}}{\gamma_{n} x_{n}}=\gamma_{n}^{-1}\left(x_{n}^{-1}+\eta_{2 n}\right) .
$$

Note that if $x_{0} \neq 0$ and $y_{0} \neq 0$, then $x_{n} \neq 0, y_{n} \neq 0 ; \forall n \in Z_{0+}$ and the above equations are well-posed. The use of the inverses sometimes facilitates the calculation for several stages ahead due to the nonlinear forms of (1), which makes their inverses have a linear evolution, $[4,6,9,11]$. Note that the above equations can be written more compactly as follows:

$$
\left[\begin{array}{l}
x_{n+1}^{-1} \\
y_{n+1}^{-1}
\end{array}\right]=\left[\begin{array}{cc}
0 & \alpha_{n}^{-1}\left(1+\eta_{1 n} x_{n}\right) \\
\gamma_{n}^{-1} & 0
\end{array}\right]\left[\begin{array}{c}
x_{n}^{-1} \\
y_{n}^{-1}
\end{array}\right]+\left[\begin{array}{c}
0 \\
\gamma_{n}^{-1} \eta_{2 n}
\end{array}\right] ; \forall n \in \mathbf{Z}_{0+}
$$

so that along two evolution stages, one has:

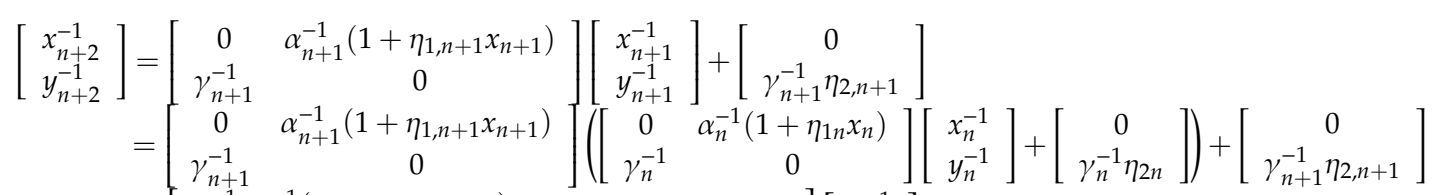

$$
\begin{aligned}
& =\left[\begin{array}{cc}
\alpha_{n+1}^{-1} \gamma_{n}^{-1}\left(1+\eta_{1, n+1} x_{n+1}\right) & 0 \\
0 & \alpha_{n}^{-1} \gamma_{n+1}^{-1}\left(1+\eta_{1 n} x_{n}\right)
\end{array}\right]\left[\begin{array}{c}
x_{n}^{-1} \\
y_{n}^{-1}
\end{array}\right] \\
& +\left[\begin{array}{cc}
0 & \alpha_{n+1}^{-1}\left(1+\eta_{1, n+1} x_{n+1}\right) \\
\gamma_{n+1}^{-1} & 0
\end{array}\right]\left[\begin{array}{c}
0 \\
\gamma_{n}^{-1} \eta_{2 n} \\
0
\end{array}\right]+\left[\begin{array}{c}
0 \\
\gamma_{n+1}^{-1} \eta_{2, n+1}
\end{array}\right] \\
& =\left[\begin{array}{cc}
\alpha_{n+1}^{-1} \gamma_{n}^{-1}\left(1+\eta_{1, n+1} x_{n+1}\right) & 0 \\
0 & \alpha_{n}^{-1} \gamma_{n+1}^{-1}\left(1+\eta_{1 n} x_{n}\right)
\end{array}\right]\left[\begin{array}{c}
x_{n}^{-1} \\
y_{n}^{-1}
\end{array}\right]+\left[\begin{array}{c}
\alpha_{n+1}^{-1} \gamma_{n}^{-1} \eta_{2 n}\left(1+\eta_{1, n+1} x_{n+1}\right) \\
\gamma_{n+1}^{-1} \eta_{2, n+1}
\end{array}\right] ; \forall n \in Z_{0+} .
\end{aligned}
$$

It turns out that for $n$ even, the matrix of the dynamics of the above inverse system is diagonal and for $n$ odd it is antidiagonal. In the following, the solution for $n$ even is addressed a follows. Define the state sequence vector of the inverse system as $x_{n}^{I}=\left(x_{n}^{-1}, y_{n}^{-1}\right)^{T}$, parameterized by:

$$
\begin{gathered}
A_{n}=A_{n}\left(x_{n}, x_{n+1}, n, n+1\right)=\left[\begin{array}{cc}
\alpha_{n+1}^{-1} \gamma_{n}^{-1}\left(1+\eta_{1, n+1} x_{n+1}\right) & 0 \\
0 & \alpha_{n}^{-1} \gamma_{n+1}^{-1}\left(1+\eta_{1 n} x_{n}\right)
\end{array}\right] \\
b_{n}=b_{n}\left(x_{n+1}, n, n+1\right)=\left[\begin{array}{c}
\alpha_{n+1}^{-1} \gamma_{n}^{-1} \eta_{2 n}\left(1+\eta_{1, n+1} x_{n+1}\right) \\
\gamma_{n+1}^{-1} \eta_{2, n+1}
\end{array}\right] .
\end{gathered}
$$

Then,

$$
x_{2 n+2}^{I}=A_{2 n} x_{2 n}^{I}+b_{2 n} ; \forall n \in Z_{0+} .
$$

By using recursive computation, one gets:

$$
x_{2 n+2}^{I}=\prod_{i=0}^{n}\left[A_{2 i}\right] x_{0}^{I}+\sum_{i=0}^{n} \prod_{j=0}^{n-i}\left[A_{2 j}\right] b_{2 i} ; \forall n \in \mathbf{Z}_{0+} .
$$

By replacing the subscript $n \rightarrow 2 n$ in (27), one gets:

$$
\begin{aligned}
x_{2 n+2}^{I} & =\left[\begin{array}{cc}
\alpha_{2 n+1}^{-1} \gamma_{2 n}^{-1}\left(1+\eta_{1,2 n+1} x_{2 n+1}\right) & 0 \\
0 & \alpha_{2 n}^{-1} \gamma_{2 n+1}^{-1}\left(1+\eta_{1,2 n} x_{2 n}\right)
\end{array}\right] x_{2 n}^{I} \\
& +\left[\begin{array}{c}
\alpha_{2 n+1}^{-1} \gamma_{2 n}^{-1} \eta_{2,2 n}\left(1+\eta_{1,2 n+1} x_{2 n+1}\right) \\
\gamma_{2 n+1}^{-1} \eta_{2,2 n+1}
\end{array}\right] ; \forall n \in \mathbf{Z}_{0+} .
\end{aligned}
$$


Assume that for some $K_{2 n}=\left[\begin{array}{ll}K_{2 n}^{11} & K_{2 n}^{12} \\ K_{2 n}^{21} & K_{2 n}^{22}\end{array}\right]$, the following constraint holds for some $\alpha_{2 n+1}, \gamma_{2 n+1}$

$$
\begin{gathered}
b_{2 n}=\left[\begin{array}{c}
\alpha_{2 n+1}^{-1} \gamma_{2 n}^{-1} \eta_{2,2 n}\left(1+\eta_{1,2 n+1} x_{2 n+1}\right) \\
\gamma_{2 n+1}^{-1} \eta_{2,2 n+1}
\end{array}\right]=\left[\begin{array}{cc}
\alpha_{2 n+1}^{-1} & 0 \\
0 & \gamma_{2 n+1}^{-1}
\end{array}\right]\left[\begin{array}{cc}
K_{2 n}^{11} & K_{2 n}^{12} \\
K_{2 n}^{21} & K_{2 n}^{22}
\end{array}\right] x_{2 n}^{I} \\
=\left[\begin{array}{cc}
\alpha_{2 n+1}^{-1} & 0 \\
0 & \gamma_{2 n+1}^{-1}
\end{array}\right]\left[\begin{array}{l}
K_{2 n}^{11} x_{2 n}^{-1}+K_{2 n}^{12} y_{2 n}^{-1} \\
K_{2 n}^{21} x_{2 n}^{-1}+K_{2 n}^{22} y_{2 n}^{-1}
\end{array}\right] ; \forall n \in \mathbf{Z}_{0+}
\end{gathered}
$$

or equivalently,

$$
\left[\begin{array}{cc}
\alpha_{2 n+1} & 0 \\
0 & \gamma_{2 n+1}
\end{array}\right] b_{2 n}=\left[\begin{array}{ll}
K_{2 n}^{11} & K_{2 n}^{12} \\
K_{2 n}^{21} & K_{2 n}^{22}
\end{array}\right] x_{2 n}^{I}=\left[\begin{array}{l}
K_{2 n}^{11} x_{2 n}^{-1}+K_{2 n}^{12} y_{2 n}^{-1} \\
K_{2 n}^{21} x_{2 n}^{-1}+K_{2 n}^{22} y_{2 n}^{-1}
\end{array}\right] ; \forall n \in \mathbf{Z}_{0+}
$$

such that the following identity holds for some prefixed suitable matrix sequence $\left\{A_{2 n}^{*}\right\}_{n=0}^{\infty}$ :

$$
A_{2 n}+\left[\begin{array}{cc}
\alpha_{2 n+1}^{-1} & 0 \\
0 & \gamma_{2 n+1}^{-1}
\end{array}\right]\left[\begin{array}{cc}
K_{2 n}^{11} & K_{2 n}^{12} \\
K_{2 n}^{21} & K_{2 n}^{22}
\end{array}\right]=A_{2 n}^{*} ; \forall n \in Z_{0+}
$$

whose convergence abscissa sequence is $\left\{\rho_{2 n}\right\}_{n=0}^{\infty} \in\left(0, \rho_{2 n}^{*}\right)$ subject to the constraint $\lim _{n \rightarrow \infty} \inf _{n} \prod_{n=0}^{\infty}\left[\rho_{2 n}^{*}\right]>0$, which avoids the uncontrolled, unbounded growing of the mosquito population, which is equivalent to the asymptotic convergence to zero of the larvae and adult populations in an infinite number of evolution stages.

Note that $\left\{A_{2 n}^{*}\right\}_{n=0}^{\infty}$ defines the suited evolution of the dynamics of the inverse system for the selection of the maximum progression gains $\alpha_{2 n+i}, \gamma_{2 n+i} ; i \in Z_{+}$. However, the sequence $\left\{A_{2 n}^{*}\right\}_{n=0}^{\infty}$ is not, in practice, of full design freedom. In particular, it is needed to prevent the environment against untolerated damage due to abusive amounts of insecticide drops. It also has to accommodate for the temperature (a key factor in the abundance of mosquitoes in a certain environment), larval diet supply, and density effects. Therefore, the intensity of the intervention has to take into account the seasonal issues and the control of environment damage under admissible bounds. Note also that if $\lim _{n \rightarrow \infty} \prod_{n=0}^{\infty}\left[\rho_{2 n}^{*}\right]=0$, then the state sequence of the population's inverse converges to zero so that the populations of larvae and adult diverge to infinity, which should be avoided if possible. Now, define the subsequent subsets of $Z_{0+}$ :

$$
N_{1}=\left\{n \in Z_{0+}: \rho_{2 n}>1\right\} ; N_{2}=\left\{n \in Z_{0+}: \rho_{2 n}<1\right\} ; N_{3}=\left\{n \in Z_{0+}: \rho_{2 n}=1\right\}
$$

and $N_{3} \neq \varnothing$ if $N_{1}=\varnothing$ and $2 \neq \varnothing$. Thus, the condition lim $\inf \prod_{n=0}^{N}\left[\rho_{2 n}^{*}\right]>0$ might be characterized alternatively as follows which is proven in Appendix A:

Assertion 1. $\lim _{n \rightarrow \infty} \inf \prod_{n=0}^{\infty}\left[\rho_{2 n}^{*}\right]>0$ if and only if there is a real constant $\varepsilon>0$ such that:

$$
\begin{gathered}
\prod_{n \in N_{1}}\left[\rho_{2 n}^{*}\right]-\varepsilon /\left(\prod_{n \in N_{2}}\left[\rho_{2 n}^{*}\right]\right) \geq 0 \text { if } N_{1} \neq \varnothing \\
\varepsilon \leq\left(\prod_{n \in N_{2}}\left[\rho_{2 n}^{*}\right]\right) \text { if } N_{1}=\varnothing .
\end{gathered}
$$

A worked example about population controls follows: 
Example 4. One and two stage ahead monitoring controls. The manipulation of the birth rate and the maximum progression rate sequences as external controls to govern the population growth can be programmed stage-by-stage in the life cycles of mosquitoes or several stages ahead. Assume that:

$$
\alpha_{n} \in\left[\alpha_{n}^{(1)}, \alpha_{n}^{(2)}\right], \gamma_{n} \in\left[\gamma_{n}^{(1)}, \gamma_{n}^{(2)}\right] ; \forall n \in \mathbf{Z}_{0+}
$$

with

$$
\begin{gathered}
\alpha_{n}^{(1)}=\left(1-\widetilde{\alpha}_{n}^{(2)}\right) \alpha_{n}^{0}, \alpha_{n}^{(2)}=\left(1-\widetilde{\alpha}_{n}^{(1)}\right) \alpha_{n}^{0}, \gamma_{n}^{(1)}=\left(1-\widetilde{\gamma}_{n}^{(2)}\right) \gamma_{n}^{0}, \gamma_{n}^{(2)}=\left(1-\widetilde{\gamma}_{n}^{(1)}\right) \gamma_{n}^{0} ; \\
\forall n \in Z_{0+}
\end{gathered}
$$

with $1 \geq \widetilde{\alpha}_{n}^{(2)}>\widetilde{\alpha}_{n}^{(1)} \geq 0$ and $1 \geq \widetilde{\gamma}_{n}^{(2)}>\widetilde{\gamma}_{n}^{(1)} \geq 0 ; \forall n \in Z_{0+}$ such that $\alpha_{n}^{0}$ and $\gamma_{n}^{0}$ are the control-free nominal values, which can be constant $\alpha=\alpha^{0}$ and $\gamma=\gamma^{0}$ or not. Note that such a kind of maximum saturating constraints $\widetilde{\alpha}_{n}^{(2)}, \widetilde{\gamma}_{n}^{(2)}$ are needed to avoid unsuitable, intolerable, excessive environment damage (for instance, to water lands, water reservoirs, nearby bush, agricultural camps, or plantations, wildlife etc.), for instance, due to distribution or overdoses of insecticide. The lower constraints are due to the lack of possibility of physically extinguishing the whole population of mosquitoes, except in extremely small isolated environments.

(a) One-stage-ahead minimization population monitoring: One gets from (1) that (26) holds. So, given the current values of $x_{2 n}$ and $y_{2 n}$, one concludes that their minimum values at the next stage (i.e., the respective maxima of their inverses) for $\alpha_{2 n} \in\left[\alpha_{2 n}^{(1)}, \alpha_{2 n}^{(2)}\right], \gamma_{2 n} \in\left[\gamma_{2 n}^{(1)}, \gamma_{2 n}^{(2)}\right]$ are the subsequent ones:

$$
\begin{aligned}
\min _{\alpha_{2 n} \in\left[\alpha_{2 n}^{(1)}, \alpha_{2 n}^{(2)}\right]} x_{2 n+1}\left(x_{2 n}, y_{2 n}\right)=x_{2 n+1}\left(\alpha_{2 n}^{(1)}\right)=\frac{\left(1-\widetilde{\alpha}_{2 n}^{(2)}\right) \alpha_{2 n}^{0} y_{2 n}}{1+\eta_{1,2 n} x_{2 n}} \\
\min _{\gamma_{2 n} \in\left[\gamma_{2 n}^{(1)}, \gamma_{2 n}^{(2)}\right]} y_{2 n+1}\left(x_{2 n}, y_{2 n}\right)=y_{2 n+1}\left(\gamma_{2 n}^{(1)}\right)=\frac{\left(1-\widetilde{\gamma}_{2 n}^{(2)}\right) \alpha_{2 n}^{0} x_{2 n}}{1+\eta_{2,2 n} x_{2 n}}
\end{aligned}
$$

(b) Two-stage ahead minimization population monitoring: One concludes from (30) and (31) that, given $x_{2 n}$, then

$$
\begin{gathered}
x_{2 n+2}^{I}=\left[\begin{array}{cc}
\alpha_{2 n+1}^{-1} \gamma_{2 n}^{-1}\left(1+\eta_{1,2 n+1} x_{2 n+1}\right) & 0 \\
0 & \alpha_{2 n}^{-1} \gamma_{2 n+1}^{-1}\left(1+\eta_{1,2 n} x_{2 n}\right)
\end{array}\right] x_{2 n}^{I} \\
+\left[\begin{array}{c}
\alpha_{2 n+1}^{-1} \gamma_{2 n}^{-1} \eta_{2,2 n}\left(1+\eta_{1,2 n+1} x_{2 n+1}\right) \\
\gamma_{2 n+1}^{-1} \eta_{2,2 n+1}
\end{array}\right] ; \forall n \in Z_{0+}
\end{gathered}
$$

and then, for already given $x_{2 n}$ and $x_{2 n+1}$, one has

$$
\max _{\alpha_{2 n} \in\left[\alpha_{2 n}^{(1)}, \alpha_{2 n}^{(2)}\right], \gamma_{2 n} \in\left[\gamma_{2 n}^{(1)}, \gamma_{2 n}^{(2)}\right]} x_{2 n+2}^{-1}\left(x_{2 n+1}, x_{2 n}\right)=\frac{1+\eta_{1,2 n+1} x_{2 n+1}+\eta_{2,2 n}\left(1+\eta_{1,2 n+1} x_{2 n+1}\right) x_{2 n}}{\left(1-\widetilde{\alpha}_{2 n+1}^{(2)}\right)\left(1-\widetilde{\gamma}_{2 n}^{(2)}\right) x_{2 n}} .
$$

However, note that

$$
\begin{aligned}
& \max _{\alpha_{2 n} \in\left[\alpha_{2 n}^{(1)}, \alpha_{2 n}^{(2)}\right], \gamma_{2 n} \in\left[\gamma_{2 n}^{(1)}, \gamma_{2 n}^{(2)}\right]} \max _{\alpha_{2 n} \in\left[\alpha_{2 n}^{(1)}, \alpha_{2 n}^{(2)}\right]} x_{2 n+1}^{-1}\left(x_{2 n+2}, y_{2 n}\right) \\
& =\frac{\eta_{1,2 n+1}\left(1-\widetilde{\alpha}_{2 n}^{(1)}\right) \alpha_{2 n}^{0} y_{2 n}+\left(1+\eta_{1,2 n} x_{2 n}\right)\left(1+\eta_{2,2 n}\left(1+\eta_{1,2 n+1} x_{2 n+1}\right) x_{2 n}\right)}{\left(1+\eta_{1,2 n} x_{2 n}\right)\left(1-\widetilde{\alpha}_{2 n+1}^{(2)}\right)\left(1-\widetilde{\gamma}_{2 n}^{(2)}\right) x_{2 n}} \text {. }
\end{aligned}
$$

As a result, it turns out that the minimization of $x_{2 n+2}$ given the larvae and adult populations at the stage $2 n$ is obtained via a maximization of the adult population at the stage $2 n+1$, which is not coincident with the 
one-stage-ahead minimization of such a population. One can conclude that the one-stage-ahead minimization strategy does not lead to a sustained minimization through future stages.

\section{Simulation Examples}

In this section, three numerical simulation examples illustrating some of the theoretical developments presented in the previous Sections are considered. The examples discuss the existence and location of equilibrium points, the existence of oscillatory solutions to the proposed model, and the use of controls to reduce the populations of larvae and adult mosquitoes.

\subsection{Non-Negativeness, Boundedness, and Location of Equilibrium Points}

Consider the stage-structured model (1) parameterized by the following sequences:

$$
\begin{gathered}
\alpha_{n}=5+\sin (2 \pi 0.1 n), \gamma_{n}=0.4+0.1 \cos (2 \pi 0.1 n) \\
\eta_{1 n}=0.15\left(1+e^{-0.1 n}\right), \eta_{2 n}=0.15\left(1+e^{-0.15 n}\right)
\end{gathered}
$$

with positive initial conditions given by $x_{0}=0.2$ and $y_{0}=0.1$. These sequences are generation-dependent and their order of magnitude is similar to that considered in [1]. A periodic behavior has been selected for $\alpha_{n}$ and $\gamma_{n}$ in order to mathematically describe a seasonal behavior of the birth and maximum progression rates, which is typical in the reproduction cycles of many animals [27]. Thus, these values were selected as a periodic variation around a positive value in order to account for this biological fact. According to Proposition 5, the sequences $\left\{x_{n}\right\}_{n=0}^{\infty}$ and $\left\{y_{n}\right\}_{n=0}^{\infty}$ remain non-negative and bounded with an upper-bound given by $x_{n+1} \leq \frac{\alpha_{n} \gamma_{n-1}}{\eta_{2, n-1}}$ and $y_{n+1} \leq \frac{\gamma_{n}}{\eta_{2 n}}$, respectively. This fact is illustrated in Figures 1 and 2, where the evolution of $\left\{x_{n}\right\}_{n=0}^{\infty}$ and $\left\{y_{n}\right\}_{n=0}^{\infty}$ is displayed, respectively. In these figures it can be observed that the elements of both sequences are positive at all times while remaining bounded. In addition, the upper bounds for both sequences are also depicted in Figures 1 and 2, showing that the actual value of the populations rest under their threshold. These figures support, thus, the results stated in Proposition 5. Moreover, from Figures 1 and 2 it can also be deduced that the calculated upper bounds are somewhat conservative, in the sense that the actual values for the populations are far from the upper bound. Therefore, the calculation of a tighter upper bound appears to be a remaining open problem.

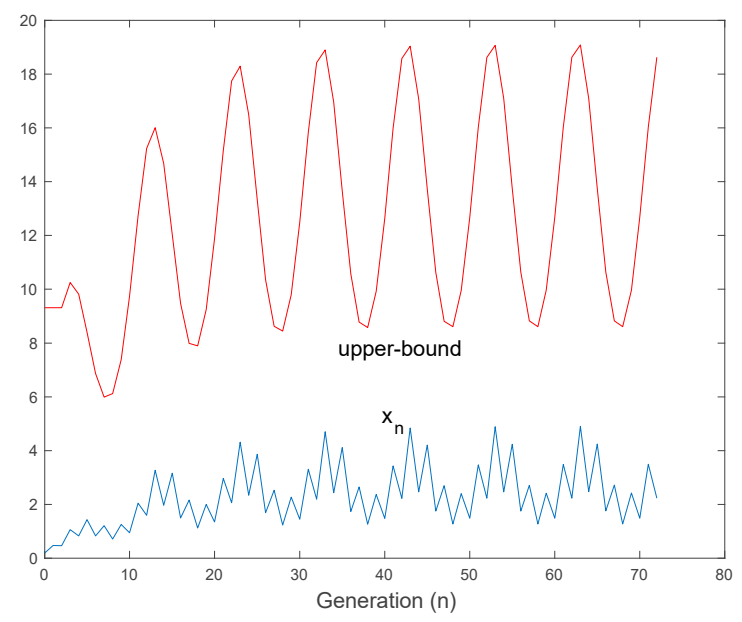

Figure 1. Evolution of the $\left\{x_{n}\right\}_{n=0}^{\infty}$ population and its calculated upper bound. 


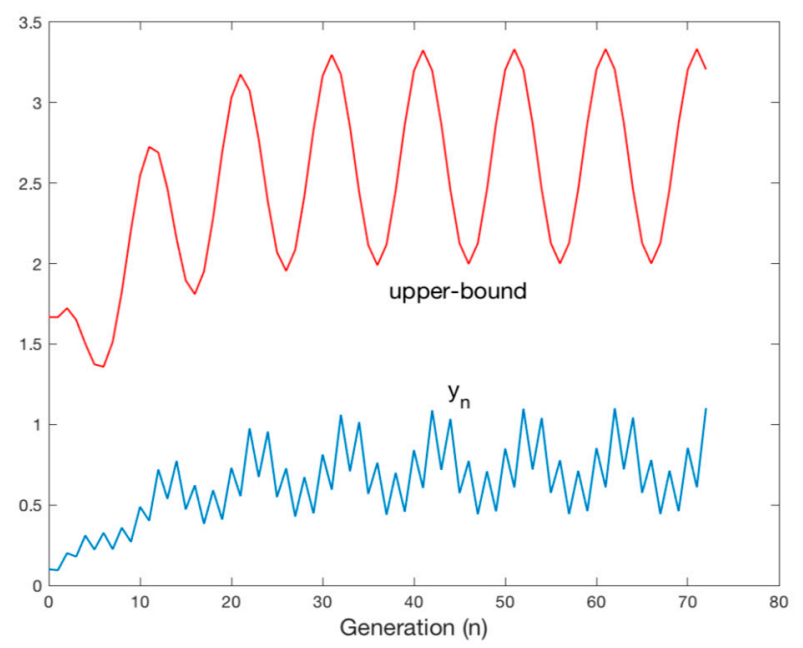

Figure 2. Evolution of the $\left\{y_{n}\right\}_{n=0}^{\infty}$ population and its calculated upper bound.

Furthermore, Proposition 7 discusses the location of the equilibrium points along with the conditions under which these exist. In order to numerically check the results claimed in Proposition 7 , we consider the following parameterization for the model sequences:

$$
\begin{gathered}
\alpha_{n}=5+e^{-0.1 n} \sin (2 \pi 0.1 n), \gamma_{n}=0.4+0.1 e^{-0.3 n} \cos (2 \pi 0.1 n), \eta_{1 n}=0.15\left(1+e^{-0.1 n}\right) \\
\eta_{2 n}=0.15\left(1+e^{-0.15 n}\right) .
\end{gathered}
$$

Now, all the sequences converge to the positive constant values:

$$
\left\{\alpha_{n}\right\}_{n=0}^{\infty} \rightarrow \alpha=5,\left\{\gamma_{n}\right\}_{n=0}^{\infty} \rightarrow \gamma=0.4,\left\{\eta_{1 n}\right\}_{n=0}^{\infty} \rightarrow \eta_{1}=0.1,\left\{\eta_{2 n}\right\}_{n=0}^{\infty} \rightarrow \eta_{2}=0.15 .
$$

Notice that under these values, the condition

$$
1<\sqrt{\alpha \gamma}=1.4142<\frac{1}{2}\left(1-\frac{\eta_{2}}{\eta_{1}}\right)+\frac{\sqrt{\left(\eta_{1}+\eta_{2}\right)^{2}+4 \eta_{1} \eta_{2}(\alpha \gamma-1)}}{2 \eta_{1}}=1.5
$$

holds. Consequently, according to Proposition 7 there exists a positive equilibrium point with coordinates:

$$
x_{e 1}=\frac{\sqrt{\left(\eta_{1}+\eta_{2}\right)^{2}+4 \eta_{1} \eta_{2}(\alpha \gamma-1)}-\left(\eta_{1}+\eta_{2}\right)}{2 \eta_{1} \eta_{2}}=\frac{10}{3}
$$

which is locally asymptotically stable. The following Figure 3 depicts the evolution of both populations with the above considered parameterization.

The final values for both populations obtained from simulation are given by $x_{\text {end }}=3.3327$ and $y_{\text {end }}=0.889$, which are in great accordance with the theoretical values presented in Proposition 7 and calculated above. Furthermore, it can be observed that the populations are positive and remain bounded as stated in Proposition 5. Moreover, Figures 4 and 5 show the evolution of both populations for different initial conditions given by: Case 1: $x_{0}=3$ and $y_{0}=1$; Case 2: $x_{0}=4$ and $y_{0}=3$; and Case 3: $x_{0}=1$ and $y_{0}=0.2$. As it is shown in Figures 4 and 5, all the trajectories converge to the same equilibrium point, which is locally asymptotically stable, as it is claimed in Proposition 7(iii). 


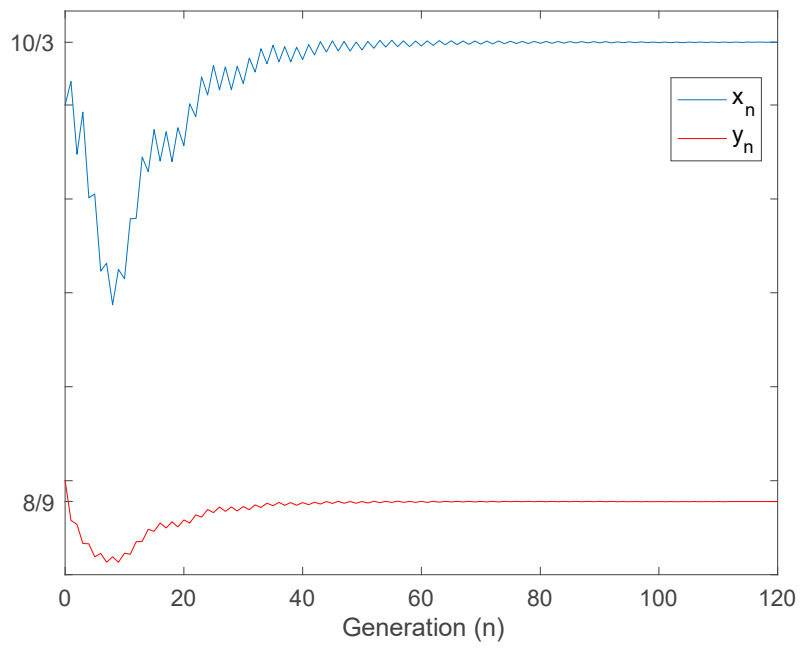

Figure 3. Evolution of $\left\{x_{n}\right\}_{n=0}^{\infty}$ and $\left\{y_{n}\right\}_{n=0}^{\infty}$ populations.

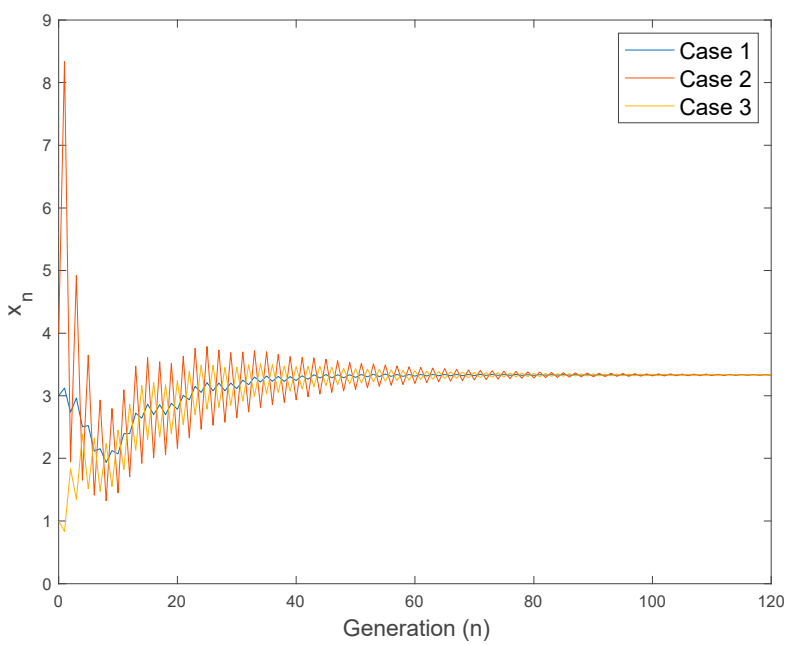

Figure 4. Evolution of $\left\{x_{n}\right\}_{n=0}^{\infty}$ for different initial conditions.

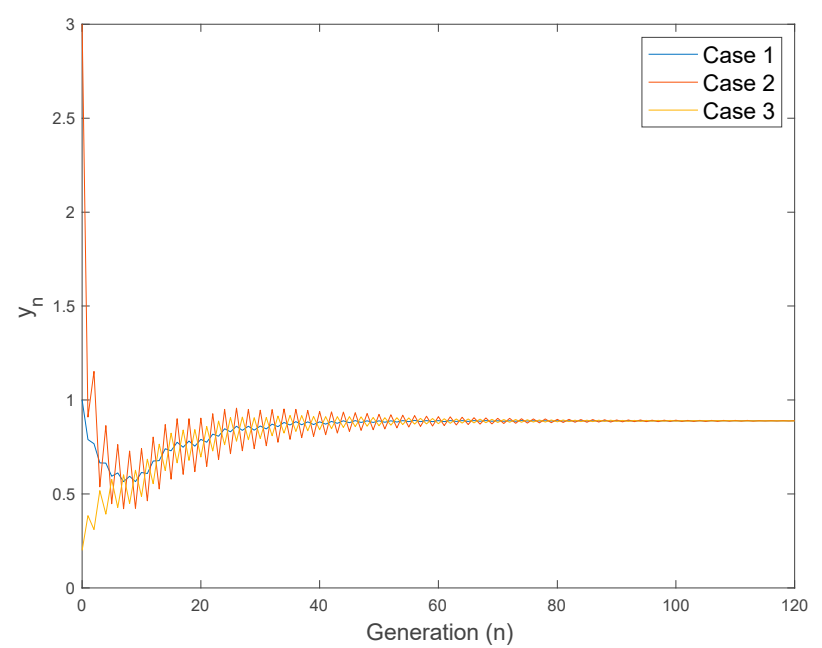

Figure 5. Evolution of $\left\{y_{n}\right\}_{n=0}^{\infty}$ for different initial conditions. 
Finally, we now change the value of $\left\{\gamma_{n}\right\}_{n=0}^{\infty}$ to $\gamma_{n}=0.2+0.1 e^{-0.3 n} \cos (2 \pi 0.1 n)$ so that the condition $\alpha \gamma=1$ holds. The initial conditions are $x_{0}=3$ and $y_{0}=1$. The Figure 6 displays the evolution of both populations in this case.

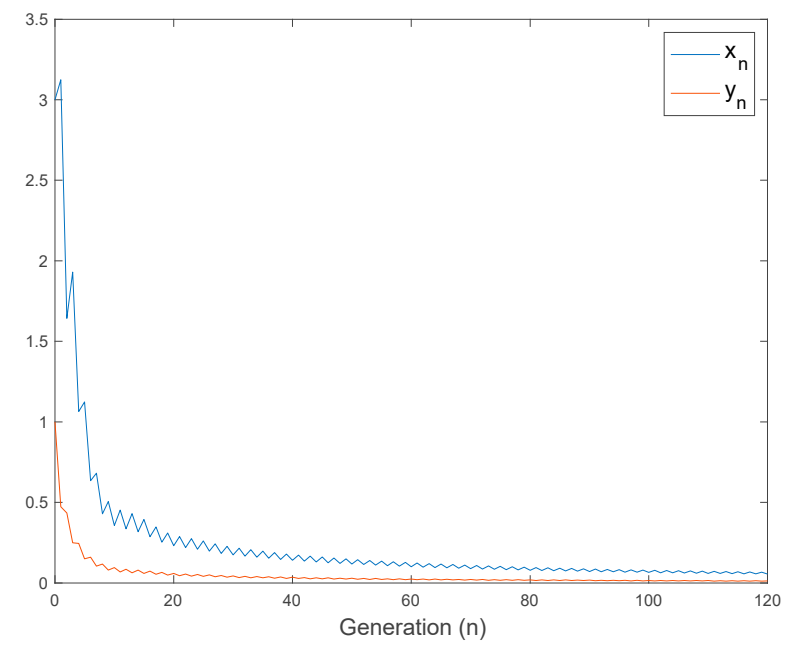

Figure 6. Evolution of $\left\{x_{n}\right\}_{n=0}^{\infty}$ and $\left\{y_{n}\right\}_{n=0}^{\infty}$ populations when $\alpha \gamma=1$.

In Figure 6 it is shown that both populations converge to the extinction equilibrium point corresponding to $\left(x_{e}, y_{e}\right)=(0,0)$. If we now complete a simulation for a large number of generations and make a zoom on the last part of it, we obtain Figure 7. In this Figure it is shown that the trajectories do not asymptotically converge to the extinction point, but they remain close to it since this point is not locally asymptotically stable as, concluded in Proposition 7(ii). Therefore, this Section illustrates some of the theoretical results contained in Section 2 regarding the non-negativity, boundedness, and existence of equilibrium points of the stage-structured model (1).

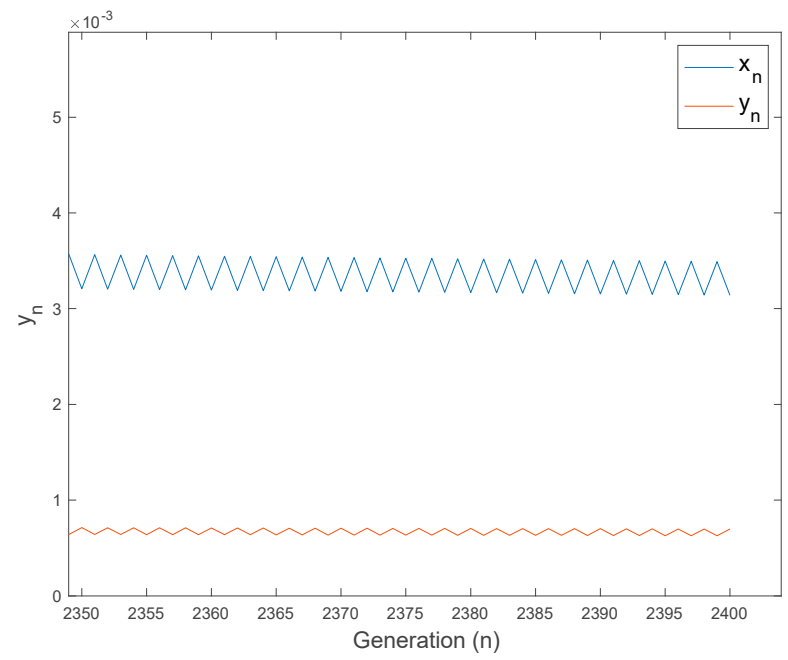

Figure 7. Zoom on $\left\{x_{n}\right\}_{n=0}^{\infty}$ and $\left\{y_{n}\right\}_{n=0}^{\infty}$ populations when $\alpha \gamma=1$ for a large number of generations.

\subsection{Oscillatory Solutions}

In this Section, a numerical example concerning the oscillatory character of the solution of the stage-structured model (1) is presented. Thus, consider the sequences given by:

$$
\begin{gathered}
\alpha_{n}=5+\sin (2 \pi 0.1 n) \gamma_{n}=k_{2 n} g_{n}=1.5+0.6 \cos (2 \pi 0.1 n), \\
\eta_{1 n}=0.15\left(1+e^{-0.1 n}\right), \eta_{2 n}=0.15\left(1+e^{-0.15 n}\right)
\end{gathered}
$$


with $k_{2 n}=1$. The Figure 8 shows the shape of sequence $\left\{\gamma_{n}\right\}_{n=0}^{\infty}$.

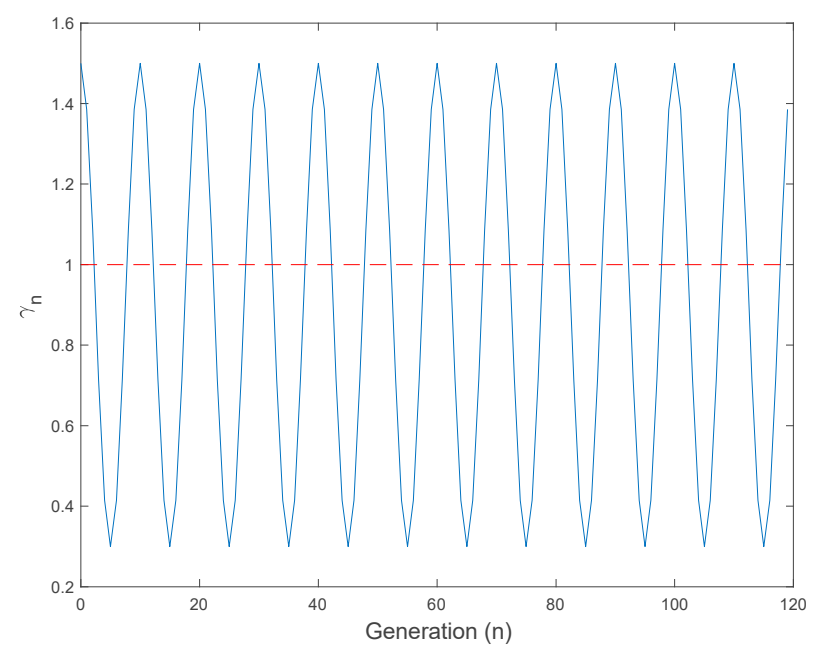

Figure 8. Evolution of the sequence $\left\{\gamma_{n}\right\}_{n=0}^{\infty}$.

From Figure 8 we can observe that there exist intervals where the sequence takes values smaller than unity, while on other intervals the values are larger than unity. Thus, it is easy to deduce that we can find two constants $k_{1}, k_{2}$ such that $\prod_{i=n}^{n+k_{1}(n)}\left[\gamma_{i}\right] \leq 1$ and $\prod_{i=n+k_{1}(n)+1}^{n+k_{2}(n)}\left[\gamma_{i}\right] \geq 1$. Consequently, we are in the position to apply Proposition 9 and conclude that the solution to the stage-structured model (1) with this parameterization will be oscillatory. Figure 9 displays the solution of system (1), corroborating the result concluded from Proposition 9.

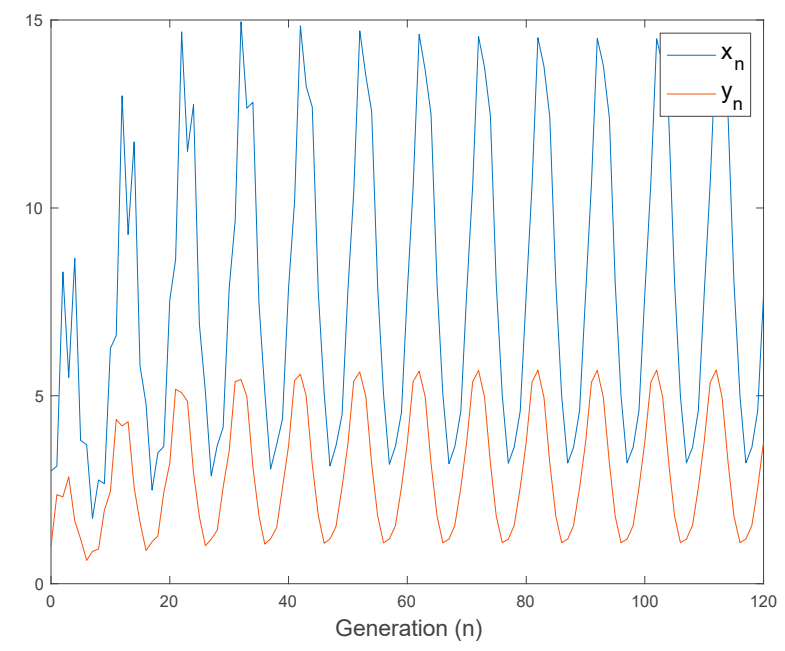

Figure 9. Oscillatory character of $\left\{x_{n}\right\}_{n=0}^{\infty}$ and $\left\{y_{n}\right\}_{n=0}^{\infty}$ populations.

\subsection{Use of Controls to Reduce the Larvae and Adult Mosquito Populations}

In this last subsection, a simulation example concerning the use of controls to reduce the larvae and adult mosquito populations is considered. Thus, consider the sequences given by:

$$
\begin{gathered}
\alpha_{n}^{0}=5+e^{-0.1 n} \sin (2 \pi 0.1 n), \gamma_{n}^{0}=0.4+0.1 e^{-0.3 n} \cos (2 \pi 0.1 n), \\
\eta_{1 n}=0.15\left(1+e^{-0.1 n}\right), \eta_{2 n}=0.15\left(1+e^{-0.15 n}\right)
\end{gathered}
$$

where $\alpha_{n}^{0}$ and $\gamma_{n}^{0}$ are referred to as the nominal control-free values. The evolution of larvae and adult mosquito populations under this parameterization are displayed in Figure 3. As it is observed 
in Figure 3, both populations converge to finite positive values, which are the equilibrium points. The objective of using controls is to reduce the values of these populations. To this end we follow Example 4 guidelines. Thus, we take $\alpha_{n} \in\left[\alpha_{n}^{(1)}, \alpha_{n}^{(2)}\right]$ and $\gamma_{n} \in\left[\gamma_{n}^{(1)}, \gamma_{n}^{(2)}\right]$ with $\alpha_{n}^{(1)}=0.6 \alpha_{n}^{(0)}$, $\alpha_{n}^{(2)}=0.9 \alpha_{n}^{(0)}, \gamma_{n}^{(1)}=0.6 \gamma_{n}^{(0)}$ and $\gamma_{n}^{(2)}=0.9 \gamma_{n}^{(0)}$. In particular in this simulation example the constant values of $\alpha_{n}=0.8 \alpha_{n}^{(0)}$ and $\gamma_{n}=0.8 \gamma_{n}^{(0)}$ are employed. Figure 10 compares the control-free evolution of populations with the controlled case.

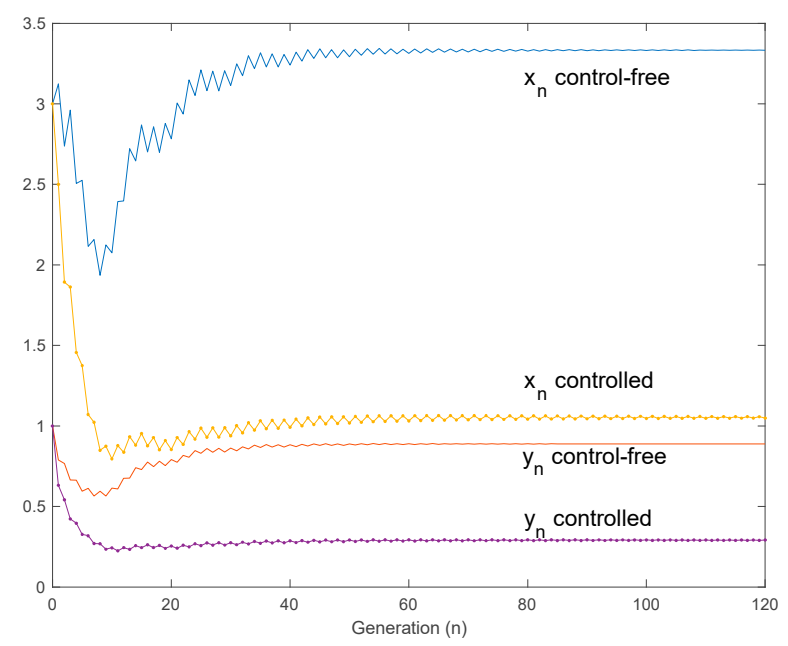

Figure 10. Comparison between the control-free and the controlled evolution of larvae and adult mosquito populations.

As it is observed in Figure 10, the control of the birth and maximum progression rate sequences allows for reducing the number of larvae and adult mosquitos, since the equilibrium point attained by the model is given by lower values than with the control-free situation. Furthermore, assume that the natural dynamics of the system for nominal parameters given by $\alpha_{n}^{0} \rightarrow \alpha^{0}$ and $\gamma_{n}^{0} \rightarrow \gamma^{0}$ make the system converge to the equilibrium point given by

$$
x_{e}^{0}=\frac{\sqrt{\left(\eta_{1}+\eta_{2}\right)^{2}+4 \eta_{1} \eta_{2}\left(\alpha^{0} \gamma^{0}-1\right)}-\left(\eta_{1}+\eta_{2}\right)}{2 \eta_{1} \eta_{2}} y_{e}^{0}=\frac{\gamma x_{e}^{0}}{1+\eta_{2} x_{e}^{0}}
$$

so that the achieved values are not biologically acceptable. We would like to reduce them to the values $x_{e}<x_{e}^{0}$ and $y_{e}<y_{e}^{0}$. To this end, we would calculate the necessary values of the birth and progression rates $\alpha, \gamma$ from the above equations, so as to get the desired new equilibrium point $\left(x_{e}, y_{e}\right)$ as:

$$
\begin{gathered}
\gamma=\frac{y_{e}\left(1+\eta_{2} x_{e}\right)}{x_{e}} \\
\alpha=\frac{1}{\gamma}\left(\frac{\left(2 \eta_{1} \eta_{2} x_{e}+\left(\eta_{1}+\eta_{2}\right)\right)^{2}-\left(\eta_{1}+\eta_{2}\right)^{2}}{4 \eta_{1} \eta_{2}}+1\right)=\frac{x_{e}}{y_{e}\left(1+\eta_{2} x_{e}\right)}\left(\frac{\left(2 \eta_{1} \eta_{2} x_{e}+\left(\eta_{1}+\eta_{2}\right)\right)^{2}-\left(\eta_{1}+\eta_{2}\right)^{2}}{4 \eta_{1} \eta_{2}}+1\right) .
\end{gathered}
$$

The last step is to calculate the amount of insecticide that must be sprayed in the atmosphere to achieve these birth and progression rates. The relationship between the amount of insecticide and the attained values for the rates depends on the spraying method and other environmental characteristics. Once the amount of insecticide is calculated, the effect of overdose and its environmental impact can be specifically considered. In this way, the formulation developed in Section 3 allows for designing feasible values for these sequences. Overall, the presented numerical examples corroborate some of the theoretical results discussed in the previous Sections 2 and 3. 


\section{Conclusions}

In this paper, the mathematical formulation of a stage-structured model of larvae and adult mosquito populations has been presented. The model is described by time-varying parameters in order to account for their potentially seasonal dependence. This situation is accepted to be of practical importance due to the typical seasonal behavior of reproduction cycles in many animal populations. The work also investigated the conditions under which the model remains non-negative, possesses attainable equilibrium points, and is bounded and/or oscillatory. These conditions are derived in terms of the sequences parameterizing the system or in the terms of its asymptotic limits, when they exist. Consequently, the paper provides useful conditions to determine when equilibrium points exist and where they are located. The value of equilibrium points inform of the steady-state larvae and mosquito populations. When these values are not acceptable for environmental or human reasons, the paper proposes the way to change the birth and progression rates in order to perform control on them. This information is necessary to calculate the amount of insecticide that should be sprayed. It has also to be taken into account the seasonal temperature, the diet stocks for larvae, and the local density effects in order to accommodate the saturated values of the maximum progression rates to be used in the control feedback implementation. Worked illustrative examples have been developed. On the other hand, further numerical simulation examples corroborate the described theoretical results.

Author Contributions: Conceptualization, M.D.L.S., conceptualization, M.D.L.S.; methodology, M.D.L.S. and A.I.; software, A.I.; validation, M.D.L.S., A.I. and A.J.G.; formal analysis, M.D.L.S.; investigation, M.D.L.S.; resources, M.D.L.S., A.I. and A.J.G.; data curation, A.I. and A.J.G.; writing-original draft preparation, M.D.L.S. and A.I.; writing-review and editing, M.D.L.S. and A.I.; visualization, M.D.L.S., A.I. and A.J.G.; supervision, M.D.L.S.; project administration, M.D.L.S. and A.J.G.; funding acquisition, M.D.L.S. and A.J.G.

Funding: This research has been cofounded by the Spanish Government and the European Regional Development Fund for Grant RTI2018-094336-B-I00 (MCIU/AEI/FEDER, UE) and by the Basque Government for Grant IT1207-19.

Acknowledgments: The authors thank the referees for their useful comments and suggestions.

Conflicts of Interest: The authors declare no conflicts of interest.

\section{Appendix A. Mathematical Proofs}

Proof of Proposition 1. Note that $K_{n} \geq 0$, if and only if $\left(1+\eta_{1 n} x_{n}\right)\left(1+\eta_{2, n-1} x_{n-1}\right) \mu x_{n}-\alpha_{n} \gamma_{n-1} x_{n-1} \geq 0$, that is, if and only if, $\left(1+\eta_{1 n} x_{n}\right) x_{n} \geq \frac{\alpha_{n} \gamma_{n-1} x_{n-1}}{\left(1+\eta_{2, n-1} x_{n-1}\right) \mu}$, equivalently if and only if

$$
\frac{x_{n}}{x_{n-1}} \geq \frac{\alpha_{n} \gamma_{n-1}}{\left(1+\eta_{1 n} x_{n}\right)\left(1+\eta_{2, n-1} x_{n-1}\right) \mu}
$$

which leads to the completeness of the proof of the first part of the proposition. The proof of the second part of the result is obvious under the change $\mu \rightarrow \mu_{n}$.

Proof of Proposition 2. Since $x_{n+1}=\frac{\mu K_{n} x_{n}}{K_{n}+(\mu-1) x_{n}}$, then $x_{n+1} \leq x_{n} ; \forall n \in Z_{+}$, if and only if $x_{n} \leq K_{n}$; $\forall n \in Z_{+}$, equivalently, if and only if $\frac{(\mu-1) \alpha_{n} \gamma_{n-1} x_{n-1}}{\left(1+\eta_{1 n} x_{n}\right)\left(1+\eta_{2, n-1} x_{n-1}\right) \mu x_{n}-\alpha_{n} \gamma_{n-1} x_{n-1}} \leq 1$, that is, if and only if, $(\mu-1) \alpha_{n} \gamma_{n-1} x_{n-1} \leq\left(1+\eta_{1 n} x_{n}\right)\left(1+\eta_{2, n-1} x_{n-1}\right) \mu x_{n}-\alpha_{n} \gamma_{n-1} x_{n-1}$, equivalently if and only if $\alpha_{n} \gamma_{n-1} \leq$ $\left(1+\eta_{1 n} x_{n}\right)\left(1+\eta_{2, n-1} x_{n-1}\right) x_{n} / x_{n-1} ; \forall n \in \mathbf{Z}_{+}$. Note that $\left\{x_{n}\right\}_{n=0}^{\infty}$ is strictly decreasing if and only if the above inequality is strict. Also, note that

$$
\frac{x_{n-1}}{x_{n}} \leq \frac{\left(1+\eta_{1 n} x_{n}\right)\left(1+\eta_{2, n-1} x_{n-1}\right)}{\alpha_{n} \gamma_{n-1}}
$$

and, if $\alpha_{n} \gamma_{n-1}>\left(1+\eta_{1 n} x_{n}\right)\left(1+\eta_{2, n-1} x_{n-1}\right)$, then $x_{n-1}<x_{n}$. Thus, a necessary condition for $x_{n+1} \leq x_{n}$; $\forall n \in Z_{+}$is that $\alpha_{n} \gamma_{n-1} \leq\left(1+\eta_{1 n} x_{n}\right)\left(1+\eta_{2, n-1} x_{n-1}\right) ; \forall n \in \mathbf{Z}_{+}$. The rest of the proof of Property (i) follows under obvious direct variations of the above part, which are omitted. Property (ii) is proven as 
follows. From the Beverton-Holt-like equation (7) under the carrying-like capacity (8) and the given intrinsic growth rate-like parameter, one concludes that:

$$
\begin{aligned}
x_{n+1}^{-1} & =\mu^{-1} x_{n}+\frac{\mu-1}{\mu} K_{n}^{-1}=\mu^{-(n-1)} x_{2}+\frac{\mu-1}{\mu} \sum_{i=2}^{n} \mu^{-n+i} K_{i}^{-1}=\mu^{-n} x_{0}+(\mu-1) \sum_{i=0}^{n} \mu^{-(n-i+1)} K_{i}^{-1} \\
& =\mu^{-(n+1)} x_{0}+(\mu-1) \mu^{-(n+1)} \sum_{i=2}^{n} \mu^{i} \frac{\left(1+\eta_{1 i} x_{i}\right)\left(1+\eta_{2, i-1} x_{i-1}\right) \mu x_{i}-\alpha_{n} \gamma_{i-1} x_{i-1}}{(\mu-1) \alpha_{i} \gamma_{i-1} x_{i} x_{i-1}} \\
& =\mu^{-(n+1)} x_{0}+\mu^{-(n+1)} \sum_{i=2}^{n} \mu^{i} \frac{\left(1+\eta_{11} x_{i}\right)\left(1+\eta_{2, i-1} x_{i-1}\right) \mu x_{i}-\alpha_{n} \gamma_{i-1} x_{i-1}}{\alpha_{i} \gamma_{i-1} x_{i} x_{i-1}} ; \forall n(\geq 2) \in \mathbf{Z}_{+}
\end{aligned}
$$

with $x_{-1}=0, x_{0}>0$ and $x_{1}=\frac{\alpha_{0} x_{0}}{1+\eta_{10} x_{0}}$. The above equations delete the first stages $n=0,1$ in order to avoid spurious calculations in the recursive equations, since $x_{-1}=0$ and $K_{0}^{-1}=+\infty$. Then, since $\mu>1$, one has the claimed result of Property (ii).

Proof of Proposition 3. From Proposition 1, $\left\{K_{n}\right\}_{n=0}^{\infty} \subset[0, \infty)$ if $\mu \geq$ $\max \left(1, \sup _{n \geq 1} \frac{\alpha_{n} \gamma_{n-1} x_{n-1}}{\left(1+\eta_{1 n} x_{n}\right)\left(1+\eta_{2, n-1} x_{n-1}\right) x_{n}}\right)$. Note that by recalculating $\alpha_{n} \gamma_{n-1} x_{n-1}$ from (8) as dependent on $K_{n}$ and from Proposition 2 that

$$
\begin{gathered}
\frac{\alpha_{n} \gamma_{n-1} x_{n-1}}{\left(1+\eta_{11} x_{n}\right)\left(1+\eta_{2, n-1} x_{n-1}\right) x_{n}}=\frac{K_{n}\left[\left(1+\eta_{1 n} x_{n}\right)\left(1+\eta_{2, n-1} x_{n-1}\right) \mu x_{n}-\alpha_{n} \gamma_{n-1} x_{n-1}\right]}{(\mu-1)\left(1+\eta_{1 n} x_{n}\right)\left(1+\eta_{2, n-1} x_{n-1}\right) x_{n}} \\
\leq \frac{x_{n}\left[\left(1+\eta_{1 n} x_{n}\right)\left(1+\eta_{2, n-1} x_{n-1}\right) \mu x_{n}-\alpha_{n} \gamma_{n-1} x_{n-1}\right]}{(\mu-1)\left(1+\eta_{1 n} x_{n}\right)\left(1+\eta_{2, n-1} x_{n-1}\right) x_{n}} \leq \frac{\mu\left(1+\eta_{1 n} x_{n}\right)\left(1+\eta_{2, n-1} x_{n-1}\right)}{(\mu-1)\left(1+\eta_{1 n} x_{n}\right)\left(1+\eta_{2, n-1} x_{n-1}\right)}=\frac{\mu}{\mu-1} .
\end{gathered}
$$

Thus, the conditions of Proposition 1 for $\left\{K_{n}\right\}_{n=0}^{\infty} \subseteq[0, \infty)$ hold if $\mu \geq \max \left(1, \frac{\mu}{\mu-1}\right)$, which holds if $\mu^{2}-2 \mu \geq 0$ with $\mu \geq 1$, that is, if $\mu \geq 2$. In addition, from Proposition $2,\left\{x_{n}\right\}_{n=0}^{\infty}$ is bounded. If the inequality is strict then the carrying capacity is positive for any $n \in Z_{+}$if $x_{0}$ is finite and non-negative.

Proof of Proposition 4. Since $\left\{\alpha_{n}\right\}_{n=0}^{\infty},\left\{\gamma_{n}\right\}_{n=0}^{\infty},\left\{\eta_{1 n}\right\}_{n=0}^{\infty}$, and $\left\{\eta_{2 n}\right\}_{n=0}^{\infty}$ are positive and bounded by hypothesis and $\left\{x_{n}\right\}_{n=0}^{\infty}$ and $\left\{y_{n}\right\}_{n=0}^{\infty}$ are non-negative if $x_{0} \geq 0$ and $y_{0} \geq 0$, note from elementary comparison of infinitesimal sequences of the same order that:

1. If $\left\{\frac{x_{2 n}}{y_{2 n}}\right\}_{n=0}^{\infty} \rightarrow 0$, then $\left\{\frac{y_{2 n+1}}{x_{2 n+1}}\right\}_{n=0}^{\infty} \rightarrow 0$, equivalently, $\left\{\frac{x_{2 n+1}}{y_{2 n+1}}\right\}_{n=0}^{\infty} \rightarrow \infty$ and then $\left\{x_{2 n}\right\}_{n=0}^{\infty} \rightarrow 0$ or $\left\{y_{2 n}\right\}_{n=0}^{\infty} \rightarrow \infty$, and $\left\{x_{2 n+1}\right\}_{n=0}^{\infty} \rightarrow \infty$ or $\left\{y_{2 n+1}\right\}_{n=0}^{\infty} \rightarrow 0$. Each of those combinations of limits either leads to a contradiction or both sequences jointly converge to zero as proven as follows:

(1a) $\quad\left\{x_{2 n}\right\}_{n=0}^{\infty} \rightarrow 0$ and $\left\{x_{2 n+1}\right\}_{n=0}^{\infty} \rightarrow \infty$ imply $\left\{y_{2 n}\right\}_{n=0}^{\infty} \rightarrow \infty$ (from the first equation of (1)) and $\left\{x_{2 n-1}\right\}_{n=1}^{\infty}$ does not diverge (from the second equation of (1)) since $\left\{\gamma_{n}\right\}_{n=0}^{\infty} ;\left\{\eta_{2 n}\right\}_{n=0}^{\infty}$ are positive and bounded. However, this contradicts $\left\{x_{2 n+1}\right\}_{n=0}^{\infty} \rightarrow \infty$. Then, $\left\{x_{2 n}\right\}_{n=0}^{\infty} \rightarrow 0$ and $\left\{x_{2 n+1}\right\}_{n=0}^{\infty} \rightarrow \infty$ is impossible if $\left\{\frac{x_{2 n}}{y_{2 n}}\right\}_{n=0}^{\infty} \rightarrow 0$.

(1b) $\quad\left\{x_{2 n}\right\}_{n=0}^{\infty} \rightarrow 0$ and $\left\{y_{2 n+1}\right\}_{n=0}^{\infty} \rightarrow 0$ imply $\left\{\left(1+\eta_{1,2 n} x_{2 n}\right) x_{2 n+1}-\alpha_{2 n} y_{2 n}\right\}_{n=0}^{\infty} \rightarrow 0$ as $n \rightarrow \infty$ from the first equation of (1) so that: 1) either $\left\{x_{2 n+1}\right\}_{n=0}^{\infty} \rightarrow 0$ and $\left\{y_{2 n}\right\}_{n=0}^{\infty} \rightarrow 0$, and then $\left\{x_{n}\right\}_{n=0}^{\infty} \rightarrow 0 \wedge\left\{y_{n}\right\}_{n=0}^{\infty} \rightarrow 0$ (so, both sequences jointly converge to zero), or 2) $\operatorname{mim}\left(\liminf _{n \rightarrow \infty} x_{2 n+1}, \liminf _{n \rightarrow \infty} y_{2 n}\right)>0$, which contradicts $\left\{x_{2 n+1}\right\}_{n=0}^{\infty} \rightarrow 0$ and $\left\{y_{2 n}\right\}_{n=0}^{\infty} \rightarrow 0$. Then, if $\left\{\frac{x_{2 n}}{y_{2 n}}\right\}_{n=0}^{\infty} \rightarrow 0$, then $\left\{x_{2 n}\right\}_{n=0}^{\infty} \rightarrow 0$ and $\left\{y_{2 n+1}\right\}_{n=0}^{\infty} \rightarrow 0$ imply that $\left\{x_{n}\right\}_{n=0}^{\infty} \rightarrow 0$ and $\left\{y_{n}\right\}_{n=0}^{\infty} \rightarrow 0$.

(1c) $\quad\left\{y_{2 n}\right\}_{n=0}^{\infty} \rightarrow \infty$ and $\left\{x_{2 n+1}\right\}_{n=0}^{\infty} \rightarrow \infty$ is impossible from the first equation of (1) so that if $\left\{y_{2 n}\right\}_{n=0}^{\infty} \rightarrow \infty$ then $\left\{x_{2 n}\right\}_{n=0}^{\infty} \rightarrow \infty$ implying the necessary constraint $\lim _{n \rightarrow \infty} \sup _{2 n+1}<\infty$. On the other hand, if $\left\{x_{2 n+1}\right\}_{n=0}^{\infty} \rightarrow \infty$, then $\lim _{n \rightarrow \infty} \sup _{2 n+1}<\infty$ from the second equation of (1) and $\left\{x_{2 n+2}\right\}_{n=0}^{\infty} \rightarrow 0$ from the first equation of (1). However, then $\left\{y_{2 n+1}\right\}_{n=0}^{\infty} \rightarrow 0$. Then, $\frac{x_{2 n+1}}{y_{2 n+1}} \rightarrow \infty$ and $\frac{y_{2 n+2}}{x_{2 n+2}} \rightarrow 0$ as $n \rightarrow \infty$ from (8). Since $\left\{x_{2 n+2}\right\}_{n=0}^{\infty} \rightarrow 0$, then $\left\{y_{2 n+2}\right\}_{n=0}^{\infty} \rightarrow 0$. 
Since $\left\{y_{2 n+1}\right\}_{n=0}^{\infty} \rightarrow 0$ and $\left\{y_{2 n+2}\right\}_{n=0}^{\infty} \rightarrow 0$, then $\left\{y_{n}\right\}_{n=0}^{\infty} \rightarrow 0$ and, from the second equation of (1), $\left\{x_{n}\right\}_{n=0}^{\infty} \rightarrow 0$.

(1d) $\quad\left\{y_{2 n}\right\}_{n=0}^{\infty} \rightarrow 0$ and $\left\{y_{2 n+1}\right\}_{n=0}^{\infty} \rightarrow 0$ implies $\left\{x_{2 n}\right\}_{n=0}^{\infty} \rightarrow 0$ from the second equation of (1) and $\left\{x_{2 n+1}\right\}_{n=0}^{\infty} \rightarrow 0$ as $n \rightarrow \infty$ from the first equation of (1). As a result, $\left\{x_{n}\right\}_{n=0}^{\infty} \rightarrow 0$ and $\left\{y_{n}\right\}_{n=0}^{\infty} \rightarrow 0$.

2. If $\left\{\frac{x_{2 n}}{y_{2 n}}\right\}_{n=0}^{\infty} \rightarrow \infty$, then $\left\{\frac{y_{2 n+1}}{x_{2 n+1}}\right\}_{n=0}^{\infty} \rightarrow \infty$, equivalently, $\left\{\frac{x_{2 n+1}}{y_{2 n+1}}\right\}_{n=0}^{\infty} \rightarrow 0$ and then $\left\{x_{2 n}\right\}_{n=0}^{\infty} \rightarrow \infty$ or $\left\{y_{2 n}\right\}_{n=0}^{\infty} \rightarrow 0$ and $\left\{x_{2 n+1}\right\}_{n=0}^{\infty} \rightarrow 0$ or $\left\{y_{2 n+1}\right\}_{n=0}^{\infty} \rightarrow \infty$. Each of those combinations either leads to a contradiction or both sequences jointly diverge to infinity as proven as follows:

(2a) $\quad\left\{x_{2 n}\right\}_{n=0}^{\infty} \rightarrow \infty$ and $\left\{x_{2 n+1}\right\}_{n=0}^{\infty} \rightarrow 0$ imply $\left\{y_{2 n+1}\right\}_{n=0}^{\infty}$ is bounded (from the second equation of (1)). Thus, from the first equation of (1) $x_{2 n+1}\left(1+\eta_{1,2 n} x_{2 n}\right)=\alpha_{2 n} y_{2 n}$. Since $\left\{x_{2 n}\right\}_{n=0}^{\infty} \rightarrow \infty$ and $\left\{x_{2 n+1}\right\}_{n=0}^{\infty} \rightarrow 0$ implies $\left\{\eta_{1 n}\right\}_{n=0}^{\infty}$ is positive and bounded, then $\left\{y_{2 n}\right\}_{n=0}^{\infty}$ is bounded and $\left\{y_{2 n+1}\right\}_{n=0}^{\infty}$ is also bounded from the second equation of (1), so that $\left\{y_{n}\right\}_{n=0}^{\infty}$ is bounded. Then, $\left\{y_{n}\right\}_{n=0}^{\infty}$ bounded and $\left\{x_{2 n}\right\}_{n=0}^{\infty} \rightarrow \infty$ imply that $\left\{x_{2 n+1}\right\}_{n=0}^{\infty} \rightarrow 0$, while $\left\{y_{n}\right\}_{n=0}^{\infty}$ bounded and $\left\{x_{2 n+1}\right\}_{n=0}^{\infty} \rightarrow 0$ imply that $\left\{x_{2 n+2}\right\}_{n=0}^{\infty}$ is bounded so that $\left\{x_{2 n}\right\}_{n=0}^{\infty} \rightarrow \infty$ is impossible. Thus, the joint conditions $\left\{x_{2 n}\right\}_{n=0}^{\infty} \rightarrow \infty$ and $\left\{x_{2 n+1}\right\}_{n=0}^{\infty} \rightarrow 0$ are impossible if $\left\{\frac{x_{2 n}}{y_{2 n}}\right\}_{n=0}^{\infty} \rightarrow \infty$.

(2b) $\quad\left\{x_{2 n}\right\}_{n=0}^{\infty} \rightarrow \infty$ and $\left\{y_{2 n+1}\right\}_{n=0}^{\infty} \rightarrow \infty$ imply $\left\{y_{2 n+1}\right\}_{n=0}^{\infty}$ bounded and, since $x_{2 n+1}\left(1+\eta_{1,2 n} x_{2 n}\right)=\alpha_{2 n} y_{2 n}$ from the first equation of (1), either $\left\{x_{2 n+1}\right\}_{n=0}^{\infty} \rightarrow 0$ and $\left\{y_{2 n}\right\}_{n=0}^{\infty}$ is bounded (which together with $\left\{y_{2 n}\right\}_{n=0}^{\infty}$ bounded implies that $\left\{y_{n}\right\}_{n=0}^{\infty}$ is bounded) or $\left\{x_{2 n+1}\right\}_{n=0}^{\infty}$ does not converge to zero so that $\left\{y_{2 n}\right\}_{n=0}^{\infty}$ is unbounded. However, this is impossible, even if $\left\{x_{2 n-1}\right\}_{n=1}^{\infty}$ is unbounded from the second equation of (13). So, $\left\{x_{2 n}\right\}_{n=0}^{\infty} \rightarrow \infty$ and $\left\{y_{2 n+1}\right\}_{n=0}^{\infty} \rightarrow \infty$ imply that $\left\{y_{n}\right\}_{n=0}^{\infty}$ is bounded, so that $\left\{y_{2 n+1}\right\}_{n=0}^{\infty} \rightarrow \infty$ is impossible, and $\left\{x_{2 n+1}\right\}_{n=0}^{\infty} \rightarrow 0$ (which implies that $\left\{y_{2 n+2}\right\}_{n=0}^{\infty} \rightarrow 0$ from the second equation of (1)) and is also bounded. Then, $\left\{x_{2 n}\right\}_{n=0}^{\infty} \rightarrow \infty$ and $\left\{y_{2 n+1}\right\}_{n=0}^{\infty} \rightarrow \infty$ are impossible if $\left\{\frac{x_{2 n}}{y_{2 n}}\right\}_{n=0}^{\infty} \rightarrow \infty$.

(2c) $\quad\left\{y_{2 n}\right\}_{n=0}^{\infty} \rightarrow 0$ and $\left\{x_{2 n+1}\right\}_{n=0}^{\infty} \rightarrow 0$ are compatible with $\left\{\frac{x_{2 n}}{y_{2 n}}\right\}_{n=0}^{\infty} \rightarrow \infty$, so with $\left\{\frac{y_{2 n+1}}{x_{2 n+1}}\right\}_{n=0}^{\infty} \rightarrow 0$.

(2d) $\quad\left\{y_{2 n}\right\}_{n=0}^{\infty} \rightarrow 0$ and $\left\{y_{2 n+1}\right\}_{n=0}^{\infty} \rightarrow \infty$ imply that $\left\{x_{2 n+1}\right\}_{n=0}^{\infty} \rightarrow 0$ and $\left\{x_{2 n}\right\}_{n=0}^{\infty} \rightarrow \infty$. Also, the second equation of (13) rewritten as $y_{2 n+1}\left(\frac{1}{x_{2 n}}+\eta_{2,2 n}\right)=\gamma_{2 n}$ leads to $\limsup _{n \rightarrow \infty}\left[\gamma_{2 n}-y_{2 n+1}\left(\frac{1}{x_{2 n}}+\eta_{2,2 n}\right)\right]=0$, implying $\limsup _{n \rightarrow \infty} y_{2 n+1}=\limsup _{n \rightarrow \infty} \frac{\gamma_{2 n}}{1 / x_{2 n}+\eta_{2,2 n}}=$ $\underset{n \rightarrow \infty}{\lim \sup } \frac{\gamma_{2 n}}{\eta_{2,2 n}}<\infty$, a contradiction to $\left\{y_{2 n+1}\right\}_{n=0}^{\infty} \rightarrow \infty$. Thus, $\left\{y_{2 n}\right\}_{n=0}^{\infty} \rightarrow 0$ and $\left\{y_{2 n+1}\right\}_{n=0}^{\infty} \rightarrow \infty$ are impossible if $\left\{\frac{x_{2 n}}{y_{2 n}}\right\}_{n=0}^{\infty} \rightarrow \infty$.

Proof of Proposition 5. Assume that there is a non-negative (for which it suffices $x_{0} \geq 0$, since $\left\{\alpha_{n}\right\}_{n=0}^{\infty}$, $\left\{\gamma_{n}\right\}_{n=0}^{\infty} ;\left\{\eta_{1 n}\right\}_{n=0}^{\infty}$, and $\left\{\eta_{2 n}\right\}_{n=0}^{\infty}$ are positive), unbounded real subsequence $\left\{x_{n_{k}}\right\}_{k=0^{\prime}}^{\infty}$ which can be chosen so that it is strictly increasing with its consecutive members $x_{n_{k}}$ and $x_{n_{k+1}}$, with $n_{k+1}=n_{k}+m\left(n_{k}\right)$ for some positive integer $m\left(n_{k}\right)$ satisfying $\frac{x_{n_{k+1}}}{x_{n_{k}}}>1$. Thus, $\frac{x_{n_{k+1}}}{x_{n_{k}}}>1$ and $x_{n_{k}}$ is arbitrarily large as $k$ is arbitrarily large, thus $\lim _{k \rightarrow \infty} x_{n_{k}}=\infty$. Then, proceed by contradiction arguments leading to:

$$
1<\frac{x_{n_{k+1}}}{x_{n_{k+1}-2}}=\frac{\alpha_{n_{k+1}-1} \gamma_{n_{k+1}-2}}{\left(1+\eta_{1 n_{k+1}-1} x_{n_{k+1}-1}\right)\left(1+\eta_{2 n_{k+1}-2} x_{n_{k+1}-2}\right)} \leq \frac{\alpha_{n_{k+1}-1} \gamma_{n_{k+1}-2}}{1+\eta_{2 n_{k+1}-2} x_{n_{k+1}-2}}
$$


and, since $\left(1+\eta_{1 n_{k+1}-1} x_{n_{k+1}-1}\right) \geq 1$ and $\lim _{k \rightarrow \infty} x_{n_{k}}=\infty$, one gets:

$$
\begin{gathered}
1<\frac{x_{n_{k+1}}}{x_{n_{k}}}=\frac{x_{n_{k+1}}}{x_{n_{k+1}-2}} \frac{x_{n_{k+1}-2}}{x_{n_{k}}} \\
=\frac{\alpha_{n_{k+1}-1} \gamma_{n_{k+1}-2}}{\left(1+\eta_{1 n_{k+1}-1} x_{n_{k+1}-1}\right)\left(1+\eta_{2 n_{k+1}-2} x_{n_{k+1}-2}\right)} \frac{\alpha_{n_{k+1}-2}}{x_{n_{k+1}}} \leq \frac{\alpha_{n_{k+1}-1} \gamma_{n_{k+1}-2}}{1+\eta_{2 n_{k+1}-2} x_{n_{k+1}-2}} \frac{x_{n_{k+1}-2}}{x_{n_{k}}}
\end{gathered}
$$

and

$$
1 \leq \lim _{n \rightarrow \infty} \inf \frac{x_{n_{k+1}}}{x_{n_{k+1}-2}} \leq \limsup _{n \rightarrow \infty} \frac{\alpha_{n-k(n)} \gamma_{n-k(n)-1}}{1+\eta_{2, n-k(n)-1} x_{n-k(n)-1}}=0
$$

thus, a contradiction. As a result, no subsequence $\left\{x_{n_{k}}\right\}_{k=0}^{\infty}$ of $\left\{x_{n}\right\}_{n=0}^{\infty}$ is either strictly increasing or unbounded if $x_{0} \geq 0$ and one can then conclude that $\left\{x_{n}\right\}_{n=0}^{\infty}$ is bounded if $x_{0} \geq 0$ is finite. From the second equation of (1), one can also conclude that $\left\{y_{n}\right\}_{n=0}^{\infty}$ is bounded if, in addition, $y_{0} \geq 0$ is finite and the proof of the first part of the Proposition is complete. On the other hand, the second equation of (1) can be rewritten equivalently as $x_{n}=\frac{y_{n+1}}{\gamma_{n}-\eta_{2 n} y_{n+1}}$, which implies that $y_{n+1} \leq \gamma_{n} / \eta_{2 n}$ if $x_{n} \geq 0$; $\forall n \in Z_{0+}$ and then, from the first equation of (1), $x_{n+1} \leq \frac{\alpha_{n} \gamma_{n-1}}{\eta_{2, n-1}\left(1+\eta_{1 n} x_{n}\right)} \leq \frac{\alpha_{n} \gamma_{n-1}}{\eta_{2, n-1}} ; \forall n \in \mathbf{Z}_{0+}$ and the proof is complete.

Outline of Proof of Proposition 6. Calculations of the expressions $y_{n+1}=y_{n+1}\left(x_{n}\right), y_{n+1}=$ $y_{n+1}\left(y_{n}, y_{n-1}\right), x_{n+1}=x_{n+1}\left(x_{n}, x_{n-1}\right)$, and $x_{n+1}=x_{n+1}\left(y_{n}, y_{n-1}\right)$ from (1) to prove Proposition 6. The second equation of (1) is $y_{n+1}=y_{n+1}\left(x_{n}\right)=\frac{\gamma_{n} x_{n}}{1+\eta_{1 n} x_{n}}$. On the other hand, from the second equation of (1), one gets $x_{n}=x_{n}\left(y_{n+1}\right)$ as $x_{n}=\frac{y_{n+1}}{\gamma_{n}-\eta_{2 n} y_{n+1}}$, which, replaced in the first equation of (1), yields $x_{n+1}=x_{n+1}\left(y_{n+1}, y_{n}\right)$ as:

$$
x_{n+1}=\frac{\alpha_{n} y_{n}}{1+\eta_{1 n} \frac{y_{n+1}}{\gamma_{n}-\eta_{2 n} y_{n+1}}}=\frac{\left(\gamma_{n}-\eta_{2 n} y_{n+1}\right) \alpha_{n} y_{n}}{\gamma_{n}-\eta_{2 n} y_{n+1}+\eta_{1 n} y_{n+1}}=\frac{\left(\gamma_{n}-\eta_{2 n} y_{n+1}\right) \alpha_{n} y_{n}}{\gamma_{n}+\left(\eta_{1 n}-\eta_{2 n}\right) y_{n+1}} .
$$

From the direct replacement of the second equation of (1) into the first one, one gets $x_{n+1}=$ $x_{n+1}\left(x_{n}, x_{n-1}\right)$ as:

$$
x_{n+1}=\frac{\alpha_{n} \frac{\gamma_{n-1} x_{n-1}}{1+\eta_{2, n-1} x_{n-1}}}{1+\eta_{1 n} x_{n}}=\frac{\alpha_{n} \gamma_{n-1} x_{n-1}}{\left(1+\eta_{1 n} x_{n}\right)\left(1+\eta_{2, n-1} x_{n-1}\right)} .
$$

The substitution of (A1) into the second equation of (1) yields $y_{n+1}=y_{n+1}\left(y_{n}, y_{n-1}\right)$ as:

$$
\begin{aligned}
y_{n+1} & =\frac{\gamma_{n} \frac{\left(\gamma_{n-1}-\eta_{2, n-1} y_{n}\right) \alpha_{n-1} y_{n-1}}{\gamma_{n-1}+\left(\eta_{1, n-1}-\eta_{2, n-1}\right) y_{n}}}{1+\eta_{2 n} \frac{\left(\gamma_{n-1}-\eta_{2, n-1} y_{n}\right) \alpha_{n-1} y_{n-1}}{\gamma_{n-1}+\left(\eta_{1, n-1}-\eta_{2, n-1}\right) y_{n}}}=\frac{\gamma_{n}\left(\gamma_{n-1}-\eta_{2, n-1} y_{n}\right) \alpha_{n-1} y_{n-1}}{\gamma_{n-1}+\left(\eta_{1, n-1}-\eta_{2, n-1}\right) y_{n}+\eta_{2 n}\left(\gamma_{n-1}-\eta_{2, n-1} y_{n}\right) \alpha_{n-1} y_{n-1}} \\
= & \frac{\gamma_{n}\left(\gamma_{n-1}-\eta_{2, n-1} y_{n}\right) \alpha_{n-1} y_{n-1}}{\gamma_{n-1}+\left(\eta_{1, n-1}-\eta_{2, n-1}\left(1+\eta_{2 n} \alpha_{n-1} y_{n-1}\right)\right) y_{n}+\eta_{2 n} \gamma_{n-1} \alpha_{n-1} y_{n-1}}
\end{aligned}
$$

and now we replace (A3) into the denominator of (A1) to yield $x_{n+1}=x_{n+1}\left(y_{n}, y_{n-1}\right)$ as:

$$
\begin{aligned}
x_{n+1} & =\frac{\left(\gamma_{n}-\eta_{2 n} y_{n+1}\right) \alpha_{n} y_{n}}{\gamma_{n}+\left(\eta_{1 n}-\eta_{2 n}\right) \frac{\gamma_{n}\left(\gamma_{n-1}-\eta_{2, n-1} y_{n}\right) \alpha_{n-1} y_{n-1}}{\gamma_{n-1}+\left(\eta_{1, n-1}-\eta_{2, n-1}\left(1+\eta_{2 n} \alpha_{n-1} y_{n-1}\right)\right) y_{n}+\eta_{2 n} \gamma_{n-1} \alpha_{n-1} y_{n-1}}} \\
& =\frac{\left(\gamma_{n}-\eta_{2 n} y_{n+1}\right) \alpha_{n} y_{n}\left[\gamma_{n-1}+\left(\eta_{1, n-1}-\eta_{2, n-1}\left(1+\eta_{2 n} \alpha_{n-1} y_{n-1}\right)\right) y_{n}+\eta_{2 n} \gamma_{n-1} \alpha_{n-1} y_{n-1}\right]}{\gamma_{n}\left[\gamma_{n-1}+\left(\eta_{1, n-1}-\eta_{2, n-1}\left(1+\eta_{2 n} \alpha_{n-1} y_{n-1}\right)\right) y_{n}+\eta_{2 n} \gamma_{n-1} \alpha_{n-1} y_{n-1}\right]+\left(\eta_{1 n}-\eta_{2 n}\right) \gamma_{n}\left(\gamma_{n-1}-\eta_{2, n-1} y_{n}\right) \alpha_{n-1} y_{n-1}}
\end{aligned}
$$

Proof of Proposition 7. From Proposition 7, write $x_{n+1}=x_{n}=\bar{x}$ in Proposition 6, Equation (16), to get $\left(1-\frac{\alpha \gamma}{1+\left(\eta_{1}+\eta_{2}\right) \bar{x}+\eta_{1} \eta_{2} \bar{x}^{2}}\right) \bar{x}=0$, which implies $\bar{x}=0$ or $1=\frac{\alpha \gamma}{1+\left(\eta_{1}+\eta_{2}\right) \bar{x}+\eta_{1} \eta_{2} \bar{x}^{2}}$, the second one implying that $\alpha \gamma=1$ if $\bar{x}=0$. Note that $\alpha \gamma=1$ if and only if $\bar{x}=0$. As a result, either $\bar{x}=0$ and $\alpha \gamma=1$ or 
$\bar{x} \neq 0$ and $\alpha \gamma \neq 1$. If $1=\frac{\alpha \gamma}{1+\left(\eta_{1}+\eta_{2}\right) \bar{x}+\eta_{1} \eta_{2} \bar{x}^{2}}$, then equivalently, $p(\bar{x}) \equiv \eta_{1} \eta_{2} \bar{x}^{2}+\left(\eta_{1}+\eta_{2}\right) \bar{x}+1-\alpha \gamma=0$, whose roots are:

$$
x_{e 1}=\frac{\sqrt{\left(\eta_{1}+\eta_{2}\right)^{2}+4 \eta_{1} \eta_{2}(\alpha \gamma-1)}-\left(\eta_{1}+\eta_{2}\right)}{2 \eta_{1} \eta_{2}} ; x_{e 2}=-\frac{\sqrt{\left(\eta_{1}+\eta_{2}\right)^{2}+4 \eta_{1} \eta_{2}(\alpha \gamma-1)}+\left(\eta_{1}+\eta_{2}\right)}{2 \eta_{1} \eta_{2}}
$$

and only one root $x_{e 1}$ of $p(\bar{x})$ is real and non-negative provided that $\alpha \gamma \geq 1$, such that $x_{e}>0$ if and only if $\alpha \gamma>1$ and $x_{e}=0$ if and only if $\alpha \gamma=1$. Now, from the second equation of (1), one gets if $y_{n+1}=y_{e}$ and $x_{n}=x_{e}$, such that

$$
y_{e}=\frac{\gamma x_{e}}{1+\eta_{2} x_{e}}=\frac{\gamma\left(\sqrt{\left(\eta_{1}+\eta_{2}\right)^{2}+4 \eta_{1} \eta_{2}(\alpha \gamma-1)}-\eta_{1}-\eta_{2}\right)}{2 \eta_{1} \eta_{2}+\eta_{2}\left(\sqrt{\left(\eta_{1}+\eta_{2}\right)^{2}+4 \eta_{1} \eta_{2}(\alpha \gamma-1)}-\eta_{1}-\eta_{2}\right)}
$$

which is zero if $x_{e}=0$, that is, if and only if $\alpha \gamma=1$, and it is positive if $x_{e}>0$, that is, if and only if $\alpha \gamma>1$. Property (i) has been proven for $x_{e}=x_{e 1}=\bar{x}$ and the corresponding $y_{e}$.

Also, note that $\left(x_{e}, y_{e}\right)$ is a globally stable (but not necessarily asymptotically stable) equilibrium point in the sense that all the solution sequences are bounded for any non-negative initial conditions since, otherwise, Proposition 5 would be contradicted. The equilibrium point is locally asymptotically stable if the linearized dynamics around it defined by the corresponding Jacobian matrix has its modes within the complex open unit circle centered at the origin. The Jacobian matrix at the equilibrium point $\left(x_{e}, y_{e}\right)$ is, [2],

$$
J=\left[\begin{array}{cc}
-\frac{\eta_{1} x_{e}}{1+\eta_{1} x_{e}} & \frac{\alpha}{1+\eta_{1} x_{e}} \\
\frac{\gamma}{\left(1+\eta_{2} x_{e}\right)^{2}} & 0
\end{array}\right]
$$

Its characteristic polynomial is

$$
p(z)=z\left(z+\frac{\eta_{1} x_{e}}{1+\eta_{1} x_{e}}\right)-\frac{\alpha \gamma}{\left(1+\eta_{1} x_{e}\right)\left(1+\eta_{2} x_{e}\right)^{2}}=\frac{1}{1+\eta_{1} x_{e}}\left[\left(1+\eta_{1} x_{e}\right) z^{2}+\eta_{1} x_{e} z-\frac{\alpha \gamma}{\left(1+\eta_{2} x_{e}\right)^{2}}\right],
$$

whose zeros are:

$$
z_{1,2}=\frac{-\eta_{1} x_{e} \pm \sqrt{\left(\eta_{1} x_{e}\right)^{2}+4 \frac{\alpha \gamma\left(1+\eta_{1} x_{e}\right)}{\left(1+\eta_{2} x_{e}\right)^{2}}}}{2\left(1+\eta_{1} x_{e}\right)}
$$

(a) If $x_{e}=y_{e}=0$, then $\left|z_{1,2}\right|=\frac{1}{1+\eta_{2} x_{e}} \sqrt{\frac{\alpha \gamma}{1+\eta_{2} x_{e}}}=\sqrt{\alpha \gamma}<1$, if and only if $\alpha \gamma<1$, but extinction needs the constraint $\alpha \gamma=1$, so a contradiction exists, and then the zero equilibrium point is not locally asymptotically stable.

(a) If $\left(x_{e}, y_{e}\right) \neq 0$ with $x_{e}+y_{e}>0$, then $-1<z_{1}<z_{2}<1$, if and only if $z_{1}>-1$ and $z_{2}<1$ (since $\left.z_{1} \neq z_{2}\right)$, that is

$$
\frac{-\eta_{1} x_{e}-\sqrt{\left(\eta_{1} x_{e}\right)^{2}+4 \frac{\alpha \gamma\left(1+\eta_{1} x_{e}\right)}{\left(1+\eta_{2} x_{e}\right)^{2}}}}{2\left(1+\eta_{1} x_{e}\right)}>-1 ; \frac{-\eta_{1} x_{e}+\sqrt{\left(\eta_{1} x_{e}\right)^{2}+4 \frac{\alpha \gamma\left(1+\eta_{1} x_{e}\right)}{\left(1+\eta_{2} x_{e}\right)^{2}}}}{2\left(1+\eta_{1} x_{e}\right)}<1
$$

or, equivalently,

$$
\begin{aligned}
& \sqrt{\left(\eta_{1} x_{e}\right)^{2}+4 \frac{\alpha \gamma\left(1+\eta_{1} x_{e}\right)}{\left(1+\eta_{2} x_{e}\right)^{2}}}<2\left(1+\eta_{1} x_{e}\right)-\eta_{1} x_{e}=2+\eta_{1} x_{e} \\
& \sqrt{\left(\eta_{1} x_{e}\right)^{2}+4 \frac{\alpha \gamma\left(1+\eta_{1} x_{e}\right)}{\left(1+\eta_{2} x_{e}\right)^{2}}}<2\left(1+\eta_{1} x_{e}\right)+\eta_{1} x_{e}=2+3 \eta_{1} x_{e}
\end{aligned}
$$


and both constraints jointly hold if and only if the, more restrictive, first one holds

$$
\left(\eta_{1} x_{e}\right)^{2}+4 \frac{\alpha \gamma\left(1+\eta_{1} x_{e}\right)}{\left(1+\eta_{2} x_{e}\right)^{2}}<4+\left(\eta_{1} x_{e}\right)^{2}+4 \eta_{1} x_{e}
$$

equivalently, if and only if,

$$
\frac{\alpha \gamma\left(1+\eta_{1} x_{e}\right)}{\left(1+\eta_{2} x_{e}\right)^{2}}<1+\eta_{1} x_{e} \Leftrightarrow \sqrt{\alpha \gamma}<1+\eta_{2} x_{e}
$$

Since $\alpha \gamma>1$ for the nonzero equilibrium point $x_{e}=x_{e 1}$ to be non-negative, one gets that the equilibrium point is positive and locally stable if and only if:

$1<\sqrt{\alpha \gamma}<1+\frac{\sqrt{\left(\eta_{1}+\eta_{2}\right)^{2}+4 \eta_{1} \eta_{2}(\alpha \gamma-1)}-\left(\eta_{1}+\eta_{2}\right)}{2 \eta_{1}}=\frac{1}{2}\left(1-\frac{\eta_{2}}{\eta_{1}}\right)+\frac{\sqrt{\left(\eta_{1}+\eta_{2}\right)^{2}+4 \eta_{1} \eta_{2}(\alpha \gamma-1)}}{2 \eta_{1}}$

and, again, since for the positivity of the equilibrium point it is again needed that $\alpha \gamma>1$, a sufficient condition from the above constraints to guarantee the local asymptotic stability of the equilibrium together with its positivity is:

$$
\begin{aligned}
\frac{1}{2}(1- & \left.\frac{\eta_{2}}{\eta_{1}}\right)>\sqrt{\alpha \gamma}-\frac{\sqrt{\left(\eta_{1}+\eta_{2}\right)^{2}+4 \eta_{1} \eta_{2}(\alpha \gamma-1)}}{2 \eta_{1}} \\
& \geq \sqrt{\alpha \gamma}-\frac{\eta_{1}+\eta_{2}+2 \sqrt{\eta_{1} \eta_{2}} \sqrt{\alpha \gamma-1}}{2 \eta_{1}} \\
& =\sqrt{\alpha \gamma}-\frac{1}{2}-\frac{\eta_{2}}{2 \eta_{1}}-\frac{\sqrt{\eta_{1} \eta_{2}} \sqrt{\alpha \gamma-1}}{\eta_{1}}
\end{aligned}
$$

so that a necessary condition for the above sufficient condition to hold is: $1>\sqrt{\alpha \gamma}-\frac{\sqrt{\eta_{1} \eta_{2}} \sqrt{\alpha \gamma-1}}{\eta_{1}}$, since $\alpha \gamma>1$. Properties (ii) to (iv) have been proven.

Proof of Proposition 8. Note that the convergence to the zero equilibrium point of the larvae population is asymptotic at exponential rate if and only if:

$$
\frac{x_{n+1}}{x_{n}}=\frac{x_{n+1}}{x_{n-1}} \frac{x_{n-1}}{x_{n}}=\frac{\alpha_{n} \gamma_{n} \gamma_{n-1} x_{n-1}}{\left(1+\eta_{1 n} x_{n}\right)\left(1+\eta_{2, n-1} x_{n-1}\right)} \frac{\left(1+\eta_{1, n-1} x_{n-1}\right)\left(1+\eta_{2, n-2} x_{n-2}\right)}{\gamma_{n} \alpha_{n-1} \gamma_{n-2} x_{n-2}}<1 .
$$

Furthermore, extinction implies that $\alpha \gamma=1$ if those gains are constant, so that we give the subsequent double constraint for the larvae extinction:

$$
\begin{gathered}
\frac{1}{\gamma_{n}} \leq \alpha_{n}<\frac{\alpha_{n-1} \gamma_{n-2} x_{n-2}\left(1+\eta_{11} x_{n}\right)\left(1+\eta_{2, n-1} x_{n-1}\right)}{\gamma_{n-1} x_{n-1}\left(1+\eta_{1, n-1} x_{n-1}\right)\left(1+\eta_{2, n-2} x_{n-2}\right)} \leq 0 ; \forall n\left(\geq n_{0}\right) \in \mathbf{Z}_{0+,} \text { some } n_{0} \in \mathbf{Z}_{0+,} \text { and } \\
\limsup _{n \rightarrow \infty}\left(\alpha_{n}-\frac{\alpha_{n-1} \gamma_{n-2} x_{n-2}\left(1+\eta_{1 n} x_{n}\right)\left(1+\eta_{2, n-1} x_{n-1}\right)}{\gamma_{n-1} x_{n-1}\left(1+\eta_{1, n-1} x_{n-1}\right)\left(1+\eta_{2, n-2} x_{n-2}\right)}\right) \leq 0
\end{gathered}
$$

together with $\liminf _{n \rightarrow \infty}\left(\alpha_{n}-\gamma_{n}^{-1}\right) \geq 0$. Since $\frac{x_{n-2}}{x_{n-1}}>1 ; \forall n\left(\geq n_{0}\right) \in \mathbf{Z}_{0+}$ and $\left\{x_{n}\right\}_{n=0}^{\infty} \rightarrow 0$, it suffices for the first above limiting condition to hold so that the subsequent constraint holds:

$$
\limsup _{n \rightarrow \infty}\left(\alpha_{n}-\frac{\alpha_{n-1} \gamma_{n-2} x_{n-2}\left(1+\eta_{1 n} x_{n}\right)\left(1+\eta_{2, n-1} x_{n-1}\right)}{\gamma_{n-1} x_{n-1}\left(1+\eta_{1, n-1} x_{n-1}\right)\left(1+\eta_{2, n-2} x_{n-2}\right)}\right) \leq 0 .
$$

It turns out that the asymptotic extinction of the larvae also implies the extinction of the adult mosquitoes. 
Proof of Proposition 9. Note that, for any $n \in Z_{0+}$ one has the following conditions:

$\frac{x\left(n+k_{1}(n)+1\right)}{x(n)}=\prod_{i=n}^{n+k_{1}(n)}\left[g_{i}\right] \leq 1$ and $\frac{x\left(n+k_{2}(n)+1\right)}{x\left(n+k_{1}(n)+1\right)}=\prod_{i=n+k_{1}(n)+1}^{n+k_{2}(n)}\left[g_{i}\right] \geq 1$ imply that $x\left(n+k_{1}(n)+1\right) \leq x(n)$ and $x\left(n+k_{2}(n)+1\right) \geq x\left(n+k_{1}(n)+1\right)$, and $\prod_{i=n}^{n+k_{1}(n)}\left[g_{i}\right] \geq 1$ and $\prod_{i=n+k_{1}(n)+1}^{n+k_{2}(n)}\left[g_{i}\right] \leq 1$ imply that $x\left(n+k_{1}(n)+1\right) \geq x(n)$ and $x\left(n+k_{2}(n)\right)+1 \leq x\left(n+k_{1}(n)+1\right)$ and the solution is oscillatory.

The strict oscillatory case and the periodic one follow as appropriate in this particular case. Property (i) has been fully proven. Properties [(ii)-(iii)] are trivial modifications to guarantee the existence of an oscillatory solution by using the various obtained upper bounds and lower bounds of the sequences $\left\{g_{n}\right\}_{n=0}^{\infty}$.

Proof of Assertion 1. Note that, if $\lim _{N \rightarrow \infty} \inf \prod_{n=0}^{N}\left[\rho_{2 n}^{*}\right]>0$, then

$$
\begin{aligned}
\prod_{n=0}^{\infty}\left[\rho_{2 n}^{*}\right]= & \left(\prod_{n \in N_{1}}\left[\rho_{2 n}^{*}\right]\right)\left(\prod_{n \in N_{2}}\left[\rho_{2 n}^{*}\right]\right)\left(\prod_{n \in N_{3}}\left[\rho_{2 n}^{*}\right]\right)=\left(\prod_{n \in N_{1}}\left[\rho_{2 n}^{*}\right]\right)\left(\prod_{n \in N_{2}}\left[\rho_{2 n}^{*}\right]\right) \\
& \quad \operatorname{limf}_{N \rightarrow \infty} \prod_{n=0}^{N}\left[\rho_{2 n}^{*}\right] \geq \inf \left(\prod_{n \in N_{1}}\left[\rho_{2 n}^{*}\right]\right) \inf \left(\prod_{n \in N_{2}}\left[\rho_{2 n}^{*}\right]\right) \\
= & \lim _{N \rightarrow \infty} \inf \left(\prod_{n=0}^{N}\left[\rho_{2 n}^{*} \times \operatorname{in}\left(N_{1}\right)\right]\right) \liminf _{N \rightarrow \infty}\left(\prod_{n=0}^{N}\left[\rho_{2 n}^{*} \times \operatorname{in}\left(N_{2}\right)\right]\right) \geq \varepsilon .
\end{aligned}
$$

If $N_{1} \cup N_{2} \neq \varnothing$ for some $\varepsilon>0$, where the following binary indicator sequences have been used:

$$
\operatorname{in}\left(N_{1}\right)=\left\{\begin{array}{c}
1 \text { if } n \in N_{1} \\
1 / \rho_{2 n}^{*} \text { if } n \notin N_{1}
\end{array} ; \text { in }\left(N_{2}\right)=\left\{\begin{array}{c}
1 \text { if } n \in N_{2} \\
1 / \rho_{2 n}^{*} \text { if } n \notin N_{2}
\end{array} .\right.\right.
$$

Then, $\prod_{n \in N_{1}}\left[\rho_{2 n}^{*}\right]-\varepsilon /\left(\prod_{n \in N_{2}}\left[\rho_{2 n}^{*}\right]\right) \geq 0$ if $N_{1} \neq \varnothing$. Note that if $N_{2}=\varnothing$ then $N_{1} \neq \varnothing$, since $N_{1} \cup N_{2} \neq \varnothing$ and the above inequality holds trivially in the form $\prod_{n \in N_{1}}\left[\rho_{2 n}^{*}\right] \geq \varepsilon$ since $\left(\prod_{n \in N_{2}}\left[\rho_{2 n}^{*}\right]\right)=$ 1. Now, if $N_{1}=\varnothing$ then $\lim _{N \rightarrow \infty} \inf \left(\prod_{n=0}^{N}\left[\rho_{2 n}^{*} \times i n\left(N_{1}\right)\right]\right)=1$ and the above inequality becomes $\liminf _{N \rightarrow \infty}\left(\prod_{n=0}^{N}\left[\rho_{2 n}^{*} \times \operatorname{in}\left(N_{2}\right)\right]\right) \geq \varepsilon$ then $\varepsilon /\left(\prod_{n \in N_{2}}\left[\rho_{2 n}^{*}\right]\right) \leq 1$ for some $\varepsilon>0$. The sufficiency part of the assertion has been proven. The necessity follows directly since $\lim _{N \rightarrow \infty} \inf _{n} \prod_{n=0}^{N}\left[\rho_{2 n}^{*}\right]=0$ implies that either $\operatorname{card}\left(N_{1} \cup N_{3}\right)<\operatorname{card}\left(N_{2}\right)=\chi_{0}$ (that is, the infinity cardinal of a denumerable set) or $\operatorname{card}\left(N_{1}\right)=\operatorname{card}\left(N_{2}\right)=\chi_{0}$ with $\left(\prod_{n \in N_{2}}\left[\rho_{2 n}^{*}\right]\right)\left(\prod_{n \in N_{1}}\left[\rho_{2 n}^{*}\right]\right)=0$, so that $\left(\prod_{n \in N_{2}}\left[\rho_{2 n}^{*}\right]\right)=0$ if $N_{1}=\varnothing$ or $\left(\prod_{n \in N_{1}}\left[\rho_{2 n}^{*}\right]\right)\left(\prod_{n \in N_{2}}\left[\rho_{2 n}^{*}\right]\right) \geq \varepsilon$, if and only if $\varepsilon=0$ if $N_{1} \neq \varnothing$.

\section{References}

1. Li, Y.; Li, J. Stage-structured discrete-time models for interacting wild and sterile mosquitoes with BevertonHolt survivability. Math. Biosci. Eng. 2019, 16, 572-602. [CrossRef] [PubMed]

2. Fister, K.R.; McCarthy, M.L.; Oppenheimer, S.F.; Collins, C. Optimal control of insects through sterile insect release and habitat modification. Math. Biosci. 2013, 244, 201-212.

3. Flores, J.C. A mathematical model for wild and sterile species in competition: Immigration. Physica A 2003, 328, 214-224. [CrossRef]

4. Li, Y.; Li, J. Discrete-time models for releases of sterile mosquitoes with Beverton-Holt type for survivability. Richerche Di Mat. 2018, 67, 141-162. [CrossRef]

5. Li, J. Malaria model with stage-structured mosquitoes. Math. Biosci. Eng. 2011, 8, 753-768. [CrossRef]

6. Stevic, S. A short proof of the Cushing-Henson conjecture. Discret. Dyn. Nat. Soc. 2006, 2006, 1-5. [CrossRef]

7. de la Sen, M.; Alonso-Quesada, S. Control issues for the Beverton-Holt equation in ecology by locally monitoring the environment carrying capacity: Non-adaptive and adaptive cases. Appl. Math. Comput. 2009, 215, 2616-2633. [CrossRef] 
8. de la Sen, M.; Alonso-Quesada, S. Model-matching-based control of the Beverton-Holt equation in ecology. Discret. Dyn. Nat. Soc. 2008, 2008, 2616-2633. [CrossRef]

9. de la Sen, M.; Alonso-Quesada, S. A control theory point of view on Beverton-Holt equation in population dynamics and some of its generalizations. Appl. Math. Comput. 2008, 215, 464-481. [CrossRef]

10. Cushing, J.M.; Henson, S.M. A periodically forced Beverton-Holt equation. J. Differ. Equ. Appl. 2008, 8, 1119-1120. [CrossRef]

11. de la Sen, M. The environment carrying capacity is not independent of the intrinsic growth rate for subcritical spawning stock biomass in the Beverton-Holt equation. Ecol. Model. 2007, 204, 2171-2273. [CrossRef]

12. Hui, Y.; Lin, G.; Sun, Q. Oscillation threshold for a mosquito population suppression model with time delay. Math. Biosci. Eng. 2019, 16, 7362-7374. [CrossRef] [PubMed]

13. Takahasi, S.E.; Miura, Y.; Miura, T. On convergence of a recursive sequence $x_{n+1}=f\left(x_{n-1}, x_{n}\right)$. Taiwan. J. Math. 2006, 10, 631-638. [CrossRef]

14. Stevic, S. On the recursive sequence $x_{n+1}=x_{n-1} / g\left(x_{n}\right)$. Taiwan. J. Math. 2002, 6, 405-414.

15. Verma, R.; Sehgal, V.K.; Nitin, V. Computational stochastic modelling to handle the crisis occurred during community epidemic. Ann. Data. Sci. 2016, 3, 119-133. [CrossRef]

16. Iggidr, A.; Souza, M.O. State estimators for some epidemiological systems. Math. Biol. 2019, 78, $225-256$. [CrossRef]

17. Yang, H.M.; Ribas-Freitas, A.R. Biological view of vaccination described by mathematical modellings: From rubella to dengue vaccines. Math. Biosci. Eng. 2018, 16, 3185-3214.

18. Koivu-Jolma, M.; Annila, A. Epidemic as a natural process. Math. Biosci. 2018, 299, 97-102. [CrossRef]

19. Meyers, L. Contact network epidemiology: Bond percolation applied to infectious disease prediction and control. Bull. Am. Math. Soc. 2007, 44, 63-86. [CrossRef]

20. de la Sen, M. On the design of hyperstable feedback controllers for a class of parameterized nonlinearities. Two application examples for controlling epidemic models. Int. J. Environ. Res. Public Health 2019, 16, 2689.

21. de la Sen, M. Parametrical non-complex tests to evaluate partial decentralized linear-output feedback control stabilization conditions for their centralized stabilization counterparts. Appl. Sci. 2019, 9, 1739. [CrossRef]

22. Cai, L.; Ai, S.; Li, J. Dynamics of mosquitoes populations with different strategies for releasing sterile mosquitoes. SIAM J. Appl. Math. 2014, 74, 1786-1809. [CrossRef]

23. Li, J.; Yuan, Z. Modelling releases of sterile mosquitoes with different strategies. J. Biol. Dyn. 2015, 9, 1-14. [CrossRef] [PubMed]

24. Pryce, J.; Choi, L.; Malone, D. Insecticide space spraying for preventing malaria transmission. Cochrane DB. Syst. Rev. 2017, 2017, CD012689. [CrossRef]

25. Smith, D.L.; Battle, K.E.; Hay, S.I.; Barker, C.M.; Scott, T.W.; McKenzie, F.E. Ross, McDonald and a theory for the dynamics and control of mosquito-transmitted pathogens. Plos Pathogens 2012, 8, e1002588. [CrossRef]

26. Couret, J.; Dotson, E.; Benedict, M.Q. Temperature, larval diet and density effects on development rate and survival of aedes aegypti (Dipta: Culicidae). PLoS ONE 2014, 9, e87468. [CrossRef]

27. Ackleh, A.S.; Jang, S.R.J. A discrete two-staged population model: Continuous versus seasonal reproduction. J. Differ. Equ. Appl. 2007, 13, 261-274. [CrossRef]

(C) 2019 by the authors. Licensee MDPI, Basel, Switzerland. This article is an open access article distributed under the terms and conditions of the Creative Commons Attribution (CC BY) license (http://creativecommons.org/licenses/by/4.0/). 